\title{
Spin-orbit-coupling induced torque in ballistic domain walls: Equivalence of charge-pumping and nonequilibrium magnetization formalisms
}

\author{
Zhe Yuan ${ }^{1,2,3}$ and Paul J. Kelly ${ }^{2}$ \\ ${ }^{1}$ The Center for Advanced Quantum Studies and Department of Physics, Beijing Normal University, 100875 Beijing, China \\ ${ }^{2}$ Faculty of Science and Technology and MESA+ Institute for Nanotechnology, University of Twente, P.O. Box 217, \\ 7500 AE Enschede, The Netherlands \\ ${ }^{3}$ Institut für Physik, Johannes Gutenberg-Universität Mainz, 55128 Mainz, Germany
}

(Received 22 February 2016; revised manuscript received 17 May 2016; published 14 June 2016)

\begin{abstract}
To study the effect of spin-orbit coupling (SOC) on spin-transfer torque in magnetic materials, we have implemented two theoretical formalisms that can accommodate SOC. Using the "charge-pumping" formalism, we find two contributions to the out-of-plane spin-transfer torque parameter $\beta$ in ballistic Ni domain walls (DWs). For short DWs, the nonadiabatic reflection of conduction electrons caused by the rapid spatial variation of the exchange potential results in an out-of-plane torque that increases rapidly with decreasing DW length. For long DWs, the Fermi level conduction channel anisotropy that gives rise to an intrinsic DW resistance in the presence of SOC leads to a linear dependence of $\beta$ on the DW length. To understand this counterintuitive divergence of $\beta$ in the long DW limit, we use the "nonequilibrium magnetization" formalism to examine the spatially resolved spin-transfer torque. The SOC-induced out-of-plane torque in ballistic DWs is found to be quantitatively consistent with the values obtained using the charge-pumping calculations, indicating the equivalence of the two theoretical methods.
\end{abstract}

DOI: 10.1103/PhysRevB.93.224415

\section{INTRODUCTION}

An electron has an intrinsic (spin) angular momentum and associated with this a magnetic moment. When an electric current flows, it is accompanied by a flow of spin angular momentum. For nonmagnetic (NM) materials like copper, the current of electrons with spin in a particular direction (e.g., "up") is compensated by an equal current of electrons with spin in the opposite direction ("down"), so there is no net flow of spin angular momentum. In a ferromagnetic (FM) material with an unequal number of spin-up and spin-down electrons, there is a flow of spin angular momentum, but this only has observable consequences when translational symmetry is broken. This happens, for example, at an interface with a NM metal where spin angular moment is injected into the NM metal leading to "spin accumulation" [1]. It also happens when the magnetization direction depends on the position in space, as in a domain wall (DW) where there is a continuous transition of the magnetization direction between two domains in which it is entirely collinear (up or down for the $180^{\circ}$ DW sketched in Fig. 1). In this case, spin angular momentum is transported by an electric current from one region of space to another where it leads to an imbalance and tends to realign the angular momentum and magnetization direction of both regions of space. This phenomenon is called "spin-transfer torque" (STT) [2-4] and it forms the basis for writing information in magnetic random access memories [5,6] or for microwave frequency STT oscillators where the injected spin forces a magnetization to precess with $\mathrm{GHz}$ frequency $[3,7,8]$. Passage of a spin-polarized current can also cause a domain wall to move. This is the principle behind a new form of shift register called "racetrack memory" $[9,10]$.

The STT was first predicted based upon the conservation of spin angular momentum; a loss of spin current, $\nabla \cdot \mathbf{j}_{s}$, corresponds to a torque $-d \mathbf{s} / d t$ exerted on the local magnetization $[2,3]$. Various theoretical methods were proposed to compute
STTs with realistic electronic structures [11-14], and a number of these were implemented with first-principles electronic structure calculations [13-16]. Not all are suitable for studying the effect of spin-orbit coupling (SOC) on STT though [17]. The spin-orbit interaction couples the electron spin to its orbital motion, and the STT exerted on a local magnetization can be larger than the maximum spin angular momentum that can be transferred from conduction electrons, i.e., an amount of $\hbar$ per electron. It was recently found that STTs arising from SOC can be more efficient in driving magnetization switching, forcing oscillation, or moving magnetic DWs [18].

Two quite distinct theoretical formalisms have been proposed to calculate the STT without assuming spin angular momentum conservation. The method proposed by the Austin group [19] is to calculate the STT in terms of the exchange interaction between the local magnetization and a nonequilibrium magnetization generated by the current. We will refer to this as the nonequilibrium magnetization (NEM) scheme. The effect of SOC is explicitly included in the Hamiltonian that is used to determine the current-induced nonequilibrium magnetization. The NEM scheme has been applied to calculate STT in spin valves [13], magnetic tunnel junctions [20], and ferromagnet/normal metal bilayers [21-23]. The other method is to consider the charge current pumped by a time varying magnetization. By making use of Onsager reciprocity relations, this can be used to derive the STT [24,25]. The charge pumping formalism is also applicable when SOC is included in the Hamiltonian.

In this paper, we study the out-of-plane STT in ballistic DWs, taking nickel as an example. While numerical values of these torques have been reported in the diffusive regime for real materials using realistic electronic structures [26,27], its physical origin remains unclear [28]. Experimental observations are usually interpreted by comparing the measured velocities of current-driven DWs with the results of micromagnetic simulations, a procedure that is not straightforward. For instance, 


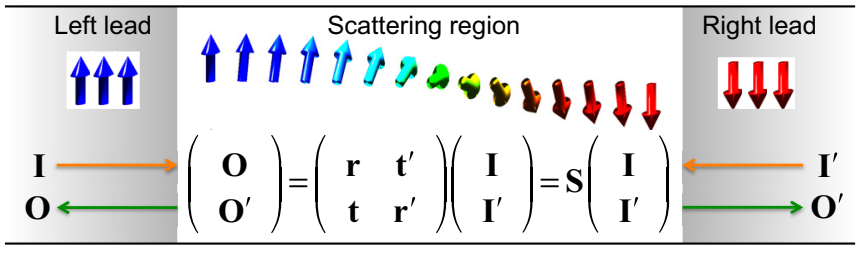

FIG. 1. Schematic illustration of the scattering theory. The scattering region consists of a $180^{\circ}$ Walker-profile DW sandwiched between semiinfinite collinearly magnetized crystalline leads. Incoming, $\mathbf{I}\left(\mathbf{I}^{\prime}\right)$, and outgoing, $\mathbf{O}\left(\mathbf{O}^{\prime}\right)$, states in the left (right) lead are connected by the scattering matrix $\mathbf{S}$ which is made up of reflection $\mathbf{r}\left(\mathbf{r}^{\prime}\right)$ and transmission $\mathbf{t}\left(\mathbf{t}^{\prime}\right)$ matrices.

the Gilbert damping must be accurately taken into account in simulations [29] but its form in noncollinear magnetizations is still the subject of discussion [30]. By implementing both the charge pumping and the NEM formalisms using firstprinciples scattering theory $[31,32]$ and using them to calculate the out-of-plane STT in ballistic DWs, we demonstrate the quantitative equivalence of the two computational schemes. The STTs obtained in ballistic DWs can be understood in terms of the scattering of electrons by the noncollinear magnetization as characterized by the DW resistance (DWR). For very short DWs, the nonadiabatic reflection of conduction electrons by the large magnetization gradients gives rise to a relatively large DWR and out-of-plane torque. In the long DW limit, the DWR is dominated by the length-independent intrinsic DWR $[33,34]$ that results from an anisotropy in the distribution of conducting channels induced by SOC in combination with the noncollinear magnetization. Electron reflection due to the intrinsic DWR gives rise to an out-of-plane torque parameter that scales linearly with the DW length. We calculate the amplitude of this torque using the two different methods and obtain values in good quantitative agreement with each other.

The rest of this paper is organized as follows. The chargepumping formalism is outlined in Sec. II followed by the results of calculations for the DWR and out-of-plane STT parameter. We present the details of the NEM scheme in Sec. III and verify the implementation for a spin valve system benchmarked in the literature; in the absence of SOC we can compare to STTs calculated using spin conservation $[13,14]$. In Sec. IV, we use the NEM scheme to calculate spatially resolved STTs in ballistic nickel DWs. The out-of-plane component quantitatively agrees with the values obtained in Sec. II using the charge-pumping formalism. Some conclusions are drawn in Sec. V.

\section{CHARGE-PUMPING FORMALISM}

\section{A. Formalism}

In this section, we briefly outline the charge-pumping formalism of Ref. [24] and how it can be combined with first-principles scattering calculations that include SOC. In the presence of an electrical current $\mathbf{j}$ with spin polarization $P$, the dynamics of a magnetization $\mathbf{M}(\mathbf{r})$ with magnitude $M_{s}$ and direction $\hat{\mathbf{M}}(\mathbf{r})$ is described by the phenomenological generalized Landau-Lifshitz-Gilbert (LLG) equation
$[4,35-38]$

$$
\begin{aligned}
\frac{d \hat{\mathbf{M}}(\mathbf{r})}{d t}= & -\gamma \hat{\mathbf{M}}(\mathbf{r}) \times \mathbf{H}_{\mathrm{eff}}(\mathbf{r}) \\
& +\hat{\mathbf{M}}(\mathbf{r}) \times\left[\int d \mathbf{r}^{\prime} \boldsymbol{\alpha}\left(\mathbf{r}, \mathbf{r}^{\prime}\right) \cdot \frac{d \hat{\mathbf{M}}\left(\mathbf{r}^{\prime}\right)}{d t}\right] \\
& -[1-\beta \hat{\mathbf{M}}(\mathbf{r}) \times]\left(\mathbf{v}_{\mathbf{s}} \cdot \nabla\right) \hat{\mathbf{M}}(\mathbf{r}),
\end{aligned}
$$

where $\mathbf{H}_{\mathrm{eff}}$ is the effective magnetic field, $\gamma=g \mu_{B} / \hbar$ is the gyromagnetic ratio expressed in terms of the Landé $g$ factor and Bohr magneton $\mu_{B}$, and $\mathbf{v}_{s}=g \mu_{B} P \mathbf{j} /\left(2 e M_{s}\right)$ is an effective velocity. In this paper, we use the following conventions. Electrons flow from the left lead to the right lead along $\hat{z}$ and the charge current density $\mathbf{j}=-|j| \hat{z}$. The electron charge is negative, $e=-|e|$. The current polarization $P$ in ballistic Ni is found to be negative since the minority-spin electrons have a larger state density at the Fermi energy than the majority-spin electrons and contribute more to the Sharvin conductance.

In Eq. (1), $\boldsymbol{\alpha}\left(\mathbf{r}, \mathbf{r}^{\prime}\right)$ and $\beta$ are the Gilbert damping and the outof-plane STT parameters, respectively. In principle, the Gilbert damping in a DW depends not only on the magnetization gradient but also on the particular mode of precession and can be calculated using first-principles scattering theory [30,39]. The effect of a nonlocal damping on DW motion [40] is beyond the scope of the present work and we will for simplicity assume a constant Gilbert damping parameter $\alpha$ in the following derivation. The out-of-plane STT parameter $\beta$ plays an important role in current-driven DW motion and is the key quantity that we calculate in this paper. It is usually assumed to be a material-dependent constant in work based upon expanding the magnetization to first-order in derivatives of the time and space variables [41]. However, in agreement with a number of other theoretical studies [28,42-45], we will see that the magnitude of the out-of-plane torque is not proportional to the magnetization gradient so that using Eq. (1) to describe the torque results in spatial variations of $\beta$ in a given magnetic texture, $\beta=\beta(\mathbf{r})$. This scenario will be confirmed later on by the position dependent STTs we will calculate for ballistic DWs requiring a fundamental reformulation of this term [46].

We consider Walker-profile [47] Bloch DWs with magnetization profile $\hat{\mathbf{M}}(z)=\left(-\tanh \frac{z-r_{w}}{\lambda_{w}},-\operatorname{sech} \frac{z-r_{w}}{\lambda_{w}}, 0\right)$ or Néel DWs with profile $\hat{\mathbf{M}}(z)=\left(\operatorname{sech} \frac{z-r_{w}}{\lambda_{w}}, 0, \tanh \frac{z-r_{w}}{\lambda_{w}}\right)$ that are centered at $r_{w}$ and have length $\lambda_{w}$. If the $\mathrm{DW}$ is displaced rigidly so that the magnetization varies in time only via the DW center, i.e.,

$$
\frac{d \hat{\mathbf{M}}}{d t}=\dot{r}_{w} \frac{d \hat{\mathbf{M}}}{d r_{w}},
$$

then the DW profile can be explicitly substituted into Eq. (1) and along the direction of the out-of-plane STT, we obtain

$$
\begin{aligned}
0= & -\gamma H_{\mathrm{ext}} \operatorname{sech}\left(\frac{z-r_{w}}{\lambda_{w}}\right)+\frac{\alpha \dot{r}_{w}}{\lambda_{w}} \operatorname{sech}\left(\frac{z-r_{w}}{\lambda_{w}}\right) \\
& -\beta(z) \frac{\hbar \gamma P j}{2 e M_{s} \lambda_{w}} \operatorname{sech}\left(\frac{z-r_{w}}{\lambda_{w}}\right) .
\end{aligned}
$$


Integrating over $z$, we are able to find a solution of the generalized LLG equation [38],

$$
\dot{r}_{w}=\frac{\gamma \lambda_{w}}{\alpha} H_{\mathrm{ext}}+\frac{\hbar \gamma P G \bar{\beta} a}{2 e A M_{s} \alpha} V .
$$

Equation (4) describes the steady state motion of a DW in response to an external field $H_{\text {ext }}$ and electrical voltage $V$ in the low current-density regime. Here $G$ is the conductance of the DW and $A$ is the cross sectional area. The parameter $\bar{\beta}$ in Eq. (4) is defined as the spatial average of $\beta(z)$ in the DW region,

$$
\bar{\beta} \equiv \frac{1}{\pi \lambda_{w}} \int \beta(z) \operatorname{sech}\left(\frac{z-r_{w}}{\lambda_{w}}\right) d z .
$$

The process reciprocal to current- or bias-driven DW motion is the "pumping" of a charge current by a moving DW $[24,25,48]$. These (reciprocal) processes can be described using coupled thermodynamic equations. We first identify two thermodynamical fluxes, the DW velocity $\dot{r}_{w}$ and charge current $I$. The conjugate forces defined by the requirement that the energy dissipation is given by the product of the flux and its conjugate force [49] are found to be $2 A M_{S} H_{\text {ext }}$ and $V$, respectively. The coupled equations can then be written as

$$
\left(\begin{array}{c}
\dot{r}_{w} \\
I
\end{array}\right)=\left(\begin{array}{ll}
\mathcal{L}_{11} & \mathcal{L}_{12} \\
\mathcal{L}_{21} & \mathcal{L}_{22}
\end{array}\right)\left(\begin{array}{c}
2 A M_{s} H_{\text {ext }} \\
V
\end{array}\right),
$$

and the coefficient $\mathcal{L}_{i j}$ characterizing how the $i$ th flux is induced by the $j$ th force can be derived as follows. Comparison of the first line of Eq. (6) with Eq. (4) yields the coefficients $\mathcal{L}_{11}=\gamma \lambda_{w} /\left(2 A M_{s} \alpha\right)$ and $\mathcal{L}_{12}=\hbar \gamma P \bar{\beta} G /\left(2 e A M_{s} \alpha\right)$. According to Ohm's law $\mathcal{L}_{22}$ is just the conductance $G$ of the DW. Reciprocity of the Onsager relations makes it possible to determine the last unknown coefficient $\mathcal{L}_{21}=\mathcal{L}_{12}$. Knowing all the coefficients in Eq. (6), the charge pumped by a DW forced to move by an external magnetic field $H_{\text {ext }}$ is found to be

$$
I=\mathcal{L}_{21} 2 A M_{s} H_{\mathrm{ext}}=\frac{\mathcal{L}_{21}}{\mathcal{L}_{11}} \dot{r}_{w}=\frac{\hbar P \bar{\beta} G}{e \lambda_{w}} \dot{r}_{w} .
$$

Using the concept of parametric pumping [50], the electrical current induced by a moving DW can alternatively be expressed in terms of the scattering matrix of the system, $\mathbf{S}=\left(\begin{array}{cc}\mathbf{r} & \mathbf{t}^{\prime} \\ \mathbf{t} & \mathbf{r}^{\prime}\end{array}\right)$, as

$$
I=\frac{e \dot{r}_{w}}{4 \pi} \operatorname{Im}\left[\operatorname{Tr}\left(\frac{\partial \mathbf{S}}{\partial r_{w}} \mathbf{S}^{\dagger} \boldsymbol{\Sigma}\right)\right]
$$

with $\mathbf{r}\left(\mathbf{r}^{\prime}\right)$ and $\mathbf{t}\left(\mathbf{t}^{\prime}\right)$ comprising matrices of reflection and transmission amplitudes for states incident from left (right) leads, respectively. The matrix $\Sigma=\left(\begin{array}{cc}1 & \mathbf{0} \\ \mathbf{0} & -\mathbf{1}^{\prime}\end{array}\right)$ consists of the unit matrices $\mathbf{1}$ and $\mathbf{1}^{\prime}$ that have the same dimensions as $\mathbf{r}$ and $\mathbf{r}^{\prime}$, respectively.

Comparing Eqs. (7) and (8), and writing the conductance in terms of the transmission matrix $\mathbf{t}$ as

$$
G=\frac{e^{2}}{h} \operatorname{Tr}\left(\mathbf{t t}^{\dagger}\right)
$$

we arrive at the required expression for $\bar{\beta}$

$$
\bar{\beta}=\frac{\lambda_{w}}{2 P \operatorname{Tr}\left(\mathbf{t t}^{\dagger}\right)} \operatorname{Im}\left[\operatorname{Tr}\left(\frac{\partial \mathbf{S}}{\partial r_{w}} \mathbf{S}^{\dagger} \boldsymbol{\Sigma}\right)\right] .
$$

Equations (9) and (10) are used in this work to directly calculate the conductance (resistance) and the out-of-plane STT parameter $\bar{\beta}$, respectively.

\section{B. Numerical details}

Our starting point is the electronic structure of bulk face-centered cubic (fcc) nickel calculated with tight-binding linearized muffin-tin orbitals (TB-LMTOs) [51,52] within the framework of density functional theory. We use the local density approximation, specifically the exchange-correlation functional parameterized by von Barth and Hedin [53], a minimal basis consisting of nine orbitals $(s, p$, and $d$ ) per spin, and sample the first Brillouin zone of the fcc lattice with $120^{3} k$ points. With the experimental lattice constant of $0.352 \mathrm{~nm}$, the charge and spin densities of collinearly magnetized fcc nickel are calculated self-consistently within the atomic spheres approximation (ASA) [54] to obtain a magnetic moment of $0.639 \mu_{B}$ per nickel atom. SOC is omitted in the self-consistent calculation since it is much smaller in energy than the band width and exchange interaction.

This electronic structure is appropriate for the semiinfinite leads. The scattering region also consists of perfectly crystalline nickel, and purely for convenience we choose the transport direction to be along the fcc [111]. The electronic structure of the scattering region is constructed by rotating the bulk atomic sphere potentials in spin space so that the local quantization axis for every atomic sphere follows the Walker magnetization profile; see Fig. 1.

We then consider the fate of each flux-normalized state $\psi_{\mu}^{I}\left(\mathbf{k}_{\|} ; E_{F}\right)$ at the Fermi energy incident from the left lead. The transmitted and reflected wave functions far away from the scattering region can be expanded in terms of all possible outgoing propagating states in the right and left leads as $\sum_{v, \mathbf{k}_{\|}^{\prime}} t_{\nu \mu}\left(\mathbf{k}_{\|}^{\prime}, \mathbf{k}_{\|}\right) \psi_{\nu}^{O^{\prime}}\left(\mathbf{k}_{\|}^{\prime} ; E_{F}\right)$ and $\sum_{\nu, \mathbf{k}_{\|}^{\prime}} r_{\nu \mu}\left(\mathbf{k}_{\|}^{\prime}, \mathbf{k}_{\|}\right) \psi_{\nu}^{O}\left(\mathbf{k}_{\|}^{\prime} ; E_{F}\right)$, respectively. The reflection and transmission coefficients $r_{\nu \mu}\left(\mathbf{k}_{\|}^{\prime}, \mathbf{k}_{\|}\right)$and $t_{\nu \mu}\left(\mathbf{k}_{\|}^{\prime}, \mathbf{k}_{\|}\right)$are determined using a "wave-function matching" scheme [55] also implemented with TB-LMTOs [31]. The same can be done for all states incident from the right lead to calculate $r_{\nu \mu}^{\prime}\left(\mathbf{k}_{\|}^{\prime}, \mathbf{k}_{\|}\right)$ and $t_{v \mu}^{\prime}\left(\mathbf{k}_{\|}^{\prime}, \mathbf{k}_{\|}\right)$and so obtain the full scattering matrix $\mathbf{S}$ explicitly.

In the absence of any disorder breaking the translational symmetry perpendicular to the transport direction, the parallel component $\mathbf{k}_{\|}$of the bulk Bloch wavevector $\mathbf{k}$ is conserved and $S_{v \mu}\left(\mathbf{k}_{\|}^{\prime}, \mathbf{k}_{\|}\right)=S_{v \mu}\left(\mathbf{k}_{\|}\right) \delta_{\mathbf{k}_{\|}^{\prime}, \mathbf{k}_{\|}}$for "ballistic" DWs. (Otherwise we could use a "lateral supercell" scheme to model disorder and allow transitions from one $\mathbf{k}_{\|}$to another $[31,56]$. It turns out that the calculated transport properties usually converge very quickly with respect to the size of the lateral supercell.) SOC is included in the transport calculations by using a Pauli Hamiltonian [32,57]. Unless otherwise stated, the two-dimensional Brillouin zone (2D BZ) is sampled using $600 \times 600 k$ points to guarantee the convergence of the calculated conductance and out-of-plane STT parameter $\bar{\beta}$. 


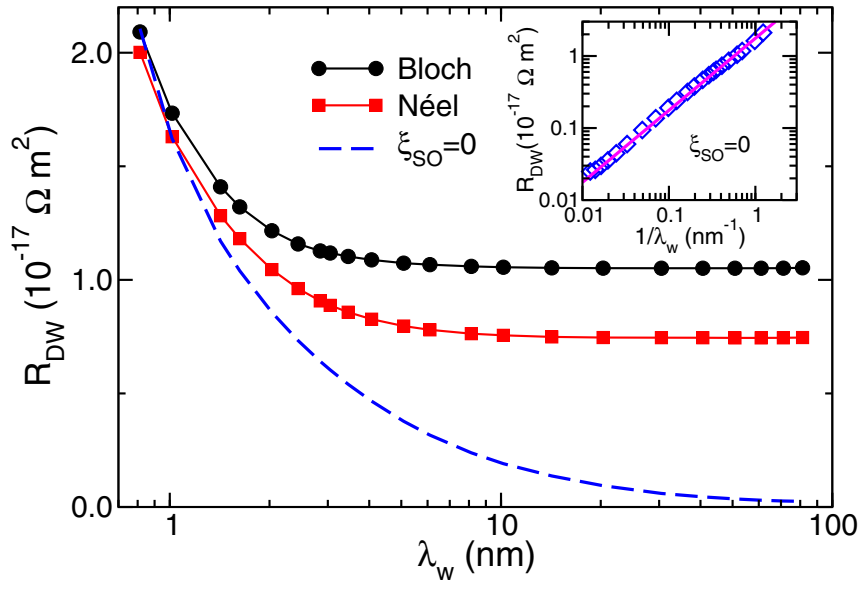

FIG. 2. DW resistance $R_{\mathrm{DW}}$ calculated for clean fcc Ni as a function of the DW length $\lambda_{w}$. For Bloch (black circles) and Néel (red squares) DWs, there are contributions to the DWR from (i) the nonadiabatic reflection of conduction electrons from short DWs $\left(\lambda_{w}<10 \mathrm{~nm}\right)$ that decreases monotonically and vanishes in the long DW limit and (ii) the conduction channel mismatch in the presence of SOC that leads to a finite saturated DWR in the long DW limit. Without SOC, there is no distinction between Bloch and Néel DWs, and only the nonadiabatic contribution is seen (dashed blue line). Inset: DWR without SOC replotted as a function of $1 / \lambda_{w}$. The solid line illustrates the linear dependence.

\section{Domain-wall resistance}

Before calculating $\bar{\beta}$ using Eq. (10), it is instructive to understand how electrons are scattered by a ballistic DW and to characterize this by the DWR $R_{\mathrm{DW}}=1 / G-1 / G_{0}$, where $G$ and $G_{0}$ are the conductances of a DW and of a bulk metal with the saturation magnetization, respectively. In particular, $G_{0}$ is the Sharvin conductance of a bulk ballistic system [58]. The DWR calculated for nickel is plotted in Fig. 2 as a function of the DW length $\lambda_{w}$. Without SOC, $R_{\mathrm{DW}}$ is large for small values of $\lambda_{w}$ because the gradient of the local magnetization is large and the conduction electrons cannot follow the rapid variation of the effective potential [59]. This nonadiabatic contribution to the DWR decreases monotonically with increasing DW length (dashed blue line) and vanishes in the long (adiabatic) limit in agreement with results found in earlier calculations [60,61]. In particular, the DWR for ballistic Ni without SOC is inversely proportional to the DW length as replotted in the inset of Fig. 2.

With SOC included, the DWR for small values of $\lambda_{w}$ is still dominated by the nonadiabatic contribution for both Bloch and Néel DWs while saturating to a finite value in the adiabatic, large- $\lambda_{w}$ limit corresponding to the so-called intrinsic DWR [33,34]. It results from a variation in the number of conduction channels at the Fermi level on rotating the magnetization direction. Figures 3(a)-3(e) show the number of conduction channels in the first BZ in the [111] direction for different values of the magnetization direction of bulk $\mathrm{Ni}$ as a function of $\mathbf{k}_{\|}$, the component of the crystal momentum perpendicular to [111]. It is equivalent to the projection of the Fermi surface onto the 2D plane perpendicular to the transport direction $[62,63]$. In ballistic systems, the crystal momentum of a propagating state is conserved and only the (a)

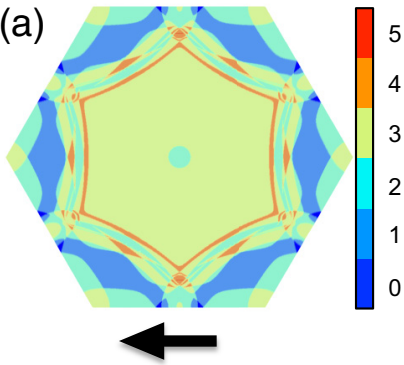

(c)

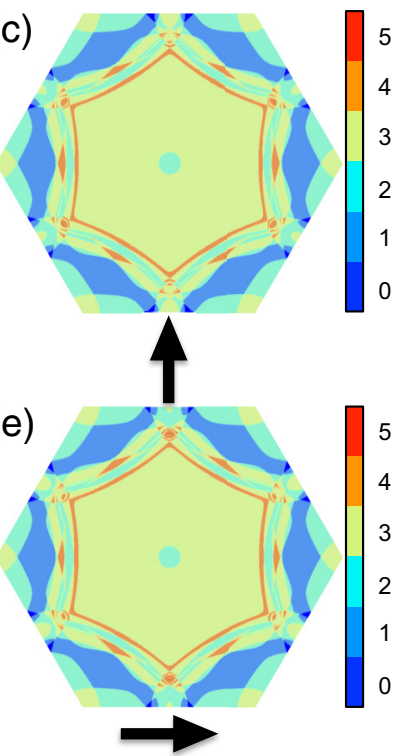

(b)

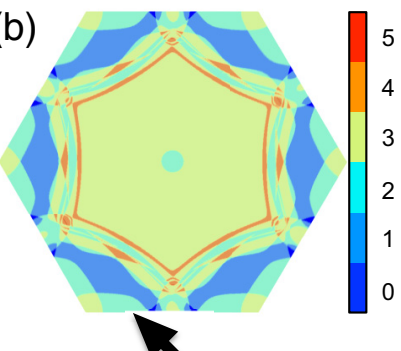

(d)

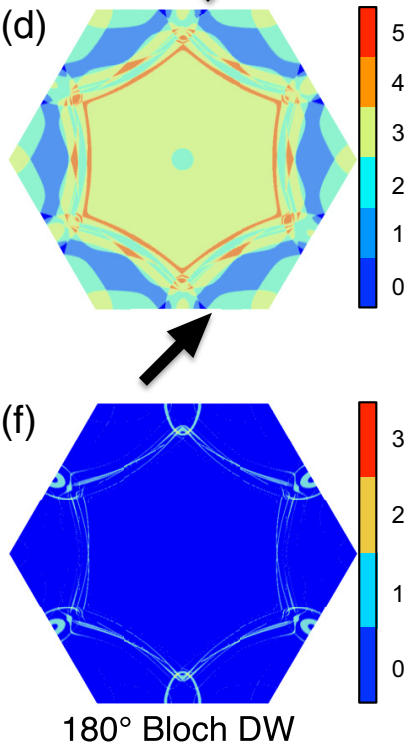

FIG. 3. (a)-(e) Calculated conduction channels at the Fermi level for fcc $\mathrm{Ni}$ along the [111] for the different magnetization orientations indicated by the arrows at the bottom of each panel. (f) The reflection probability of conduction electrons in a very long $180^{\circ}$ Bloch DW. Large reflection probabilities are found for values of $\mathbf{k}_{\|}$where the number of conduction channels depends upon the magnetization direction in (a)-(e).

propagating channels that survive for all magnetization directions contribute to the total conductance. At some $\mathbf{k}_{\|}$points the number of channels decreases as the magnetization rotates resulting in the reflection of the corresponding propagating electronic states. The total reflection in a long Bloch DW is plotted in Fig. 3(f). Large values of reflection probability are found for $\mathbf{k}_{\|}$points where the number of conduction channels varies strongly with the magnetization direction shown in Figs. 3(a)-3(e). Indeed, the intrinsic, saturated DWRs for Bloch and Néel walls can be well reproduced by counting the number of common conducting channels through the DWs. Since SOC is very weak in $3 d$ transition metals, it only slightly modifies their Fermi surfaces and the number of conduction channels for different magnetization orientations. Quantitatively, the intrinsic DWR is only $1.8 \%$ and $1.3 \%$ of the corresponding Sharvin resistance for the Bloch and Néel DWs, respectively. Note that the intrinsic DWR that is a nonlocal effect is eliminated in the diffusive regime, where spin-flip scattering and anisotropic magnetoresistance become the main mechanisms responsible for the DWR found there [64]. 


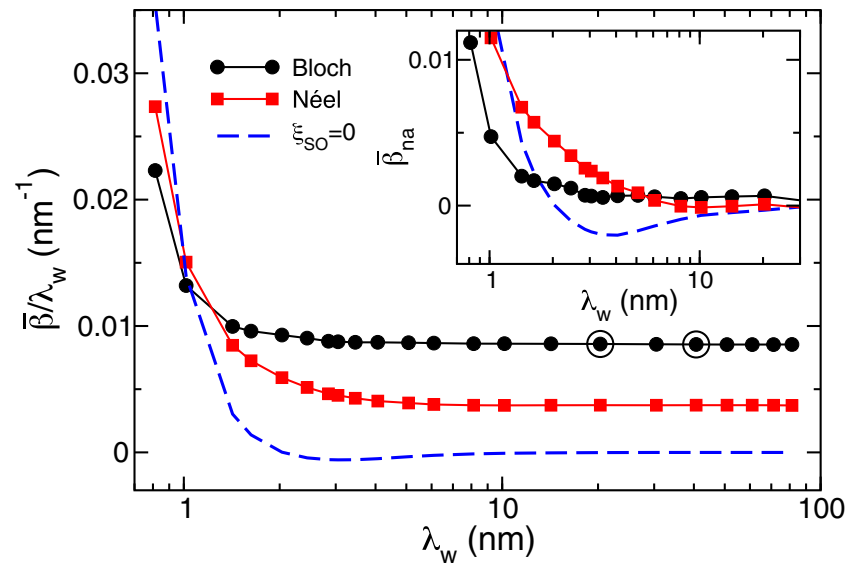

FIG. 4. Calculated out-of-plane STT parameter $\bar{\beta} / \lambda_{w}$ of clean Ni DWs as a function of the DW length $\lambda_{w} \cdot \bar{\beta} / \lambda_{w}$ saturates to a finite value for both Bloch and Néel DWs in the presence of SOC. For short DWs $\left(\lambda_{w}<10 \mathrm{~nm}\right)$, the nonadiabatic contribution to $\bar{\beta}$ increases dramatically with decreasing DW length; this increase does not depend on SOC. The large open circles denote saturated values of $\bar{\beta} / \lambda_{w}=0.0086 \mathrm{~nm}^{-1}$ for Bloch DWs with $\lambda_{w}=20$ and $40 \mathrm{~nm}$ referred to in Sec. IV. Inset: the nonadiabatic contribution $\bar{\beta}_{\text {na }}$ as a function of $\lambda_{w}$, where the contribution proportional to $\lambda_{w}$ arising from SOC has been subtracted for Bloch and Néel DWs.

\section{Out-of-plane spin-transfer torque parameter $\overline{\boldsymbol{\beta}}$}

The values of $\bar{\beta} / \lambda_{w}$ calculated using Eq. (10) are plotted in Fig. 4 as a function of the DW length $\lambda_{w}$. For both Bloch and Néel DWs, $\bar{\beta} / \lambda_{w}$ saturates to a finite value for large values of $\lambda_{w}$, indicating that $\bar{\beta}$ diverges in this adiabatic limit. The contribution that is proportional to the DW length arises from SOC; it vanishes if the SOC is switched off in the calculations as shown by the dashed blue line.

For short DWs with $\lambda_{w}<10 \mathrm{~nm}$, there is another contribution to $\bar{\beta}$ coming from the nonadiabatic reflection of conduction electrons that is not intrinsically related to SOC. This nonadiabatic contribution, $\bar{\beta}_{\text {na }}$, is plotted in the inset to Fig. 4, together with the values for Bloch and Néel DWs with the (SOC-induced) contributions proportional to $\lambda_{w}$ subtracted. $\bar{\beta}_{\text {na }}$ increases rapidly with decreasing DW length and exhibits oscillations at small values of $\lambda_{w}$. This nonadiabatic contribution to $\bar{\beta}$ has been theoretically predicted and interpreted in terms of standing waves that result from the interference of incoming and reflected electrons [42,44].

The divergent contribution arising from SOC is counterintuitive and has not been discussed in the literature. The remainder of this paper will be devoted to understanding it. To do so, we will use calculations based upon the physically transparent NEM scheme.

\section{NONEQUILIBRIUM MAGNETIZATION SCHEME}

We begin this section with a brief description of the NEM scheme proposed by Núñez and MacDonald [19,65] that can be used to calculate the spatially resolved STT $\boldsymbol{\tau}(\mathbf{r})$, and of our MTO implementation of this scheme. We illustrate it with calculations for a spin valve consisting of $\mathrm{Co}$ and $\mathrm{Cu}$ multilayers where, in the absence of SOC, the calculated STT is in good quantitative agreement with the values obtained using a method based upon spin conservation [14].

\section{A. Formalism}

In the NEM scheme, the torque exerted on a local magnetization $\mathbf{M}(\mathbf{r})$ is given by

$$
\boldsymbol{\tau}(\mathbf{r})=-\gamma \mathbf{M}(\mathbf{r}) \times \mathbf{h}^{\mathrm{ex}}(\mathbf{r}),
$$

where $\mathbf{h}^{\mathrm{ex}}(\mathbf{r})$ is the exchange field generated by the nonequilibrium magnetization $\mathbf{m}^{\text {ne }}(\mathbf{r})$ induced by a charge current. All occupied states contribute to $\mathbf{M}(\mathbf{r})$ so that direct calculation of Eq. (11) involves an integration over energy up to the Fermi energy. Since an equal and opposite torque is exerted on $\mathbf{m}^{\mathrm{ne}}(\mathbf{r})$ by the local magnetization $\mathbf{M}(\mathbf{r})$, it can be expressed as

$$
\boldsymbol{\tau}(\mathbf{r})=-\gamma \mathbf{H}^{\mathrm{ex}}(\mathbf{r}) \times \mathbf{m}^{\mathrm{ne}}(\mathbf{r}),
$$

where $\mathbf{H}^{\mathrm{ex}}(\mathbf{r})$ is the exchange field generated by the local magnetization $\mathbf{M}(\mathbf{r})[13,19,66]$. Within linear response, $\mathbf{m}^{\text {ne }}(\mathbf{r})$ is composed of contributions from propagating electronic states at the Fermi level. $\mathbf{H}^{\mathrm{ex}}(\mathbf{r})$ only depends on the equilibrium magnetization $\mathbf{M}(\mathbf{r})$ and can be readily evaluated when carrying out the self-consistent equilibrium calculations that involve calculating all occupied states. Within the ASA, evaluation of the torque can be simplified by expanding $\mathbf{H}^{\mathrm{ex}}(\mathbf{r})$ and $\mathbf{m}^{\text {ne }}(\mathbf{r})$ in spherical harmonics $Y_{l m}(\hat{\mathbf{r}})$ on site $\mathbf{R}$. On integrating over $\mathbf{r}$, we find that the torque can be decomposed into site $(\mathbf{R})$ and angular momentum $(l)$ resolved contributions as

$$
\boldsymbol{\tau}_{\mathbf{R}}=\sum_{l} \boldsymbol{\tau}_{\mathbf{R} l}=-\gamma \sum_{l} \mathbf{H}_{\mathbf{R} l}^{\mathrm{ex}} \times \mathbf{m}_{\mathbf{R} l}^{\mathrm{ne}} .
$$

Assuming that the bias $V_{b}$ applied over the scattering region is infinitesimal, $\mathbf{m}_{\mathbf{R} l}^{\text {ne }}$ can be constructed from wave functions with energy equal to the Fermi energy

$$
\begin{aligned}
\mathbf{m}_{\mathbf{R} l}^{\mathrm{ne}}=-\frac{\mu_{B}}{N_{k_{\|}}} \sum_{\mathbf{k}_{\|}} & \left(\sum_{i \in \mathcal{L}} \sum_{m}\left\langle\Psi_{\mathbf{R} l m}^{i \mathbf{k}_{\|}}|\hat{\boldsymbol{\sigma}}| \Psi_{\mathbf{R} l m}^{i \mathbf{k}_{\|}}\right\rangle\right. \\
& \left.-\sum_{j \in \mathcal{R}} \sum_{m}\left\langle\Psi_{\mathbf{R} l m}^{j \mathbf{k}_{\|}}|\hat{\boldsymbol{\sigma}}| \Psi_{\mathbf{R} l m}^{j \mathbf{k}_{\|}}\right\rangle\right) \frac{e V_{b}}{2},
\end{aligned}
$$

where $\Psi_{\mathbf{R} l m}^{i \mathbf{k}_{\|}}$and $\Psi_{\mathbf{R} l m}^{j \mathbf{k}_{\|}}$are $l m$ components of the fluxnormalized scattering wave functions $\left(|\Psi|^{2}\right.$ having the dimensions of an inverse energy) with transverse crystal momentum $\mathbf{k}_{\|}$, on site $\mathbf{R}$, incident from the left $(i \in \mathcal{L})$ and right $(j \in \mathcal{R})$ leads, respectively. Equation (14) implies that we consider both right-going electrons from the left lead and left-going holes from the right lead simultaneously [67]. Note that the bias $V_{b}$ in Eq. (14) will be eventually removed by calculating the torque per unit current density $\tau / j$, in units of $\mu_{B} /(e \mathrm{~nm})$, where $j=G V_{b} / A$ with $A$ being the cross sectional area.

The exchange field on site $\mathbf{R}$ can be decomposed in a similar fashion and $\mathbf{H}_{\mathbf{R} l}^{\mathrm{ex}}$ obtained by considering test electrons at the Fermi level with up and down spin [68],

$$
\begin{aligned}
\mathbf{H}_{\mathbf{R} l}^{\mathrm{ex}}= & -\frac{\hat{\mathbf{M}}}{4 \mu_{B}} \int d r\left\{r^{2}\left[v_{\mathbf{R} \uparrow}(r)-v_{\mathbf{R} \downarrow}(r)\right]\right. \\
& \left.\times\left[\phi_{\mathbf{R} l \uparrow}^{2}\left(\varepsilon_{\mathrm{F}}, r\right)+\phi_{\mathbf{R} l \downarrow}^{2}\left(\varepsilon_{\mathrm{F}}, r\right)\right]\right\} .
\end{aligned}
$$




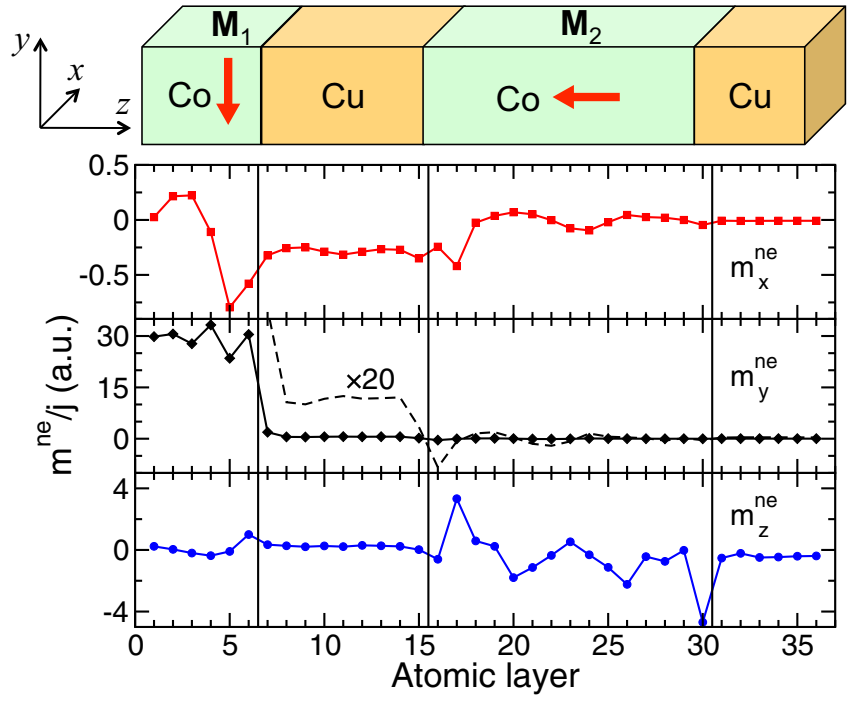

FIG. 5. Nonequilibrium magnetization calculated without SOC for the [111] oriented spin valve consisting of 9 layers of fcc $\mathrm{Cu}$ and 15 layers of $\mathrm{Co}$ sandwiched between semiinfinite $\mathrm{Co}$ and $\mathrm{Cu}$ leads shown schematically at the top. The dashed line in the central panel is increased by a factor of 20 for clarity.

Here the radial integration is carried out inside the atomic sphere on site $\mathbf{R}$ and the $l$ th partial wave $\phi_{\mathbf{R} l \sigma}\left(\varepsilon_{\mathrm{F}}, r\right)$ is obtained by solving the scalar-relativistic radial equation [69] at the energy $\varepsilon=\varepsilon_{\mathrm{F}}$ for the spin-dependent radial potential $v_{\mathbf{R} \sigma}(r)$ with $\sigma=\uparrow, \downarrow[51]$.

\section{B. Test case: $\mathrm{Co} \mid \mathrm{Cu}$ spin valve}

To verify our implementation of the NEM scheme, we consider the STTs in a system that has been studied before (without SOC) $[13,14,16], \mathrm{a} \mathrm{Co}|\mathrm{Cu}| \mathrm{Co}$ spin valve for which the spin torque has been calculated by assuming spin conservation. The spin valve is schematically shown at the top of Fig. 5. The scattering region consists of $\mathrm{Co}(6)|\mathrm{Cu}(9)| \mathrm{Co}(15) \mid \mathrm{Cu}(6)$ with the thicknesses in parantheses given in numbers of atomic layers. The left and right leads are bulk $\mathrm{Co}$ and $\mathrm{Cu}$, respectively. (In the piecewise self-consistent equilibrium calculations, the atomic sphere potentials in six layers of Co on the left and of $\mathrm{Cu}$ on the right are allowed to differ from the bulk potentials of the semiinfinite leads.) A uniform lattice constant of $3.55 \AA$ is used, and transport is along the fcc [111] with electron flow from left to right. The magnetization directions of the two ferromagnetic Co layers are chosen to be perpendicular to one other, as indicated by the thick arrows in Fig. 5. A $2400 \times 2400$ $k$ sampling of the $2 \mathrm{D} \mathrm{BZ}$ is used to obtain a well-converged out-of-plane component of the torque [66]; see the inset of Fig. 7. SOC was turned off in this test case to compare the results with those obtained with the spin conservation method [70].

The nonequilibrium magnetization arises from the difference between the nonequilibrium spin densities

$$
n_{\mathbf{R} \sigma}^{\mathrm{ne}}(\varepsilon)=\frac{1}{N_{k_{\|}}} \sum_{\mathbf{k}_{\|}} \sum_{i \in \mathcal{L}} \sum_{l, m}\left\langle\Psi_{\mathbf{R} l m \sigma}^{i \mathbf{k}_{\|}}(\varepsilon) \mid \Psi_{\mathbf{R} l m \sigma}^{i \mathbf{k}_{\|}}(\varepsilon)\right\rangle .
$$

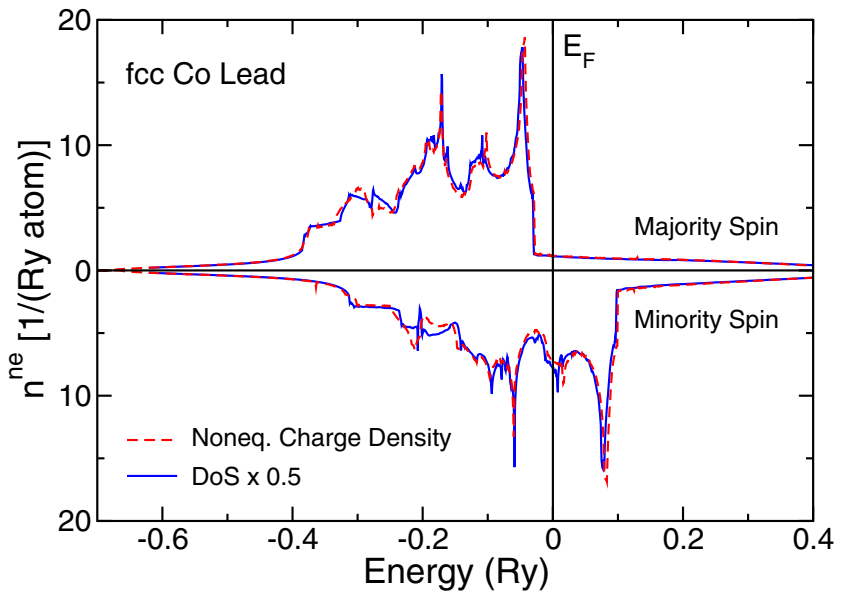

FIG. 6. Nonequilibrium spin density of a perfectly crystalline fcc Co lead calculated as a function of the energy. The density of states of bulk fcc Co is plotted for comparison.

For a perfectly crystalline fcc Co lead, we plot $n_{\mathbf{R} \sigma}^{\text {ne }}$ as a function of the energy $\varepsilon$ of the incoming electrons in Fig. 6. The nonequilibrium spin densities equal half of the total density of states (DoS) at the same energy because only electrons incident from the left are considered in Eq. (16) [holes incident from the right contribute the same amount; see Eq. (14)]. The muffin-tin orbitals used to calculate the DoS with the "bulk" LMTO code [52] are linearized in energy with $\varepsilon_{v l \sigma}$ fixed at the corresponding centers of gravity $\bar{\varepsilon}_{v l \sigma}$ of the $s, p$, and $d$ channels while the DoS is calculated using the tetrahedron method [71]. The nonequilibrium spin density $n_{\mathbf{R} \sigma}^{\mathrm{ne}}(\varepsilon)$ on the other hand is obtained in the scattering code with $\varepsilon_{v l \sigma}=\varepsilon$ and with discrete summation over $\mathbf{k}_{\|}$. These factors account for the slight differences seen in Fig. 6. At the Fermi level, minority spins contribute more nonequilibrium states, so $\mathbf{m}^{\text {ne }}$ is antiparallel to the local magnetization $\mathbf{M}$ in $\mathbf{C o}$ that is dominated by the occupied majority spin states.

The nonequilibrium magnetization $\mathbf{m}^{\text {ne }}$ generated in the $\mathrm{Co}(6)|\mathrm{Cu}(9)| \mathrm{Co}(15) \mid \mathrm{Cu}(6)$ spin valve by the electric current is plotted in Fig. 5. Since there is no disorder in either the $\mathrm{Cu}$ or Co layers, scattering only occurs at the interfaces. At a $\mathrm{Co} \mid \mathrm{Cu}$ interface, there is a large mismatch between the $\mathrm{Cu}$ and $\mathrm{Co}$ electronic structures for the minority spin channel, leading to a significant reflection of these electrons. This corresponds to a large minority-spin interface resistance [31,72] and leads to the accumulation of the minority spin density $m_{y}^{\text {ne }}$ seen in Fig. 5 antiparallel to the local magnetization direction in layer $\mathbf{M}_{1}$. The oscillations (between layers 0 and 7) are a consequence of the interference between incident and reflected waves.

The magnetizations of the two ferromagnetic layers in Fig. 5 are perpendicular to each other. The spin current transmitted through the first $\mathbf{M}_{1} \mid \mathrm{Cu}$ interface is oriented along the $-y$ direction. In this $\mathrm{Cu}$ "spacer" layer, accumulation of nonequilibrium magnetization is mostly of minority-spin electrons (along $+y$ ) injected through the $\mathrm{Co} \mid \mathrm{Cu}$ interface. There are also contributions (along $-x$ ) from multiple scattering at the two $\mathrm{Cu}$ interfaces. Without a local magnetization (and spin relaxation) in $\mathrm{Cu}$, these propagating states keep their spin polarization. The quantization axis of the $\mathbf{M}_{2}$ layer is at right 


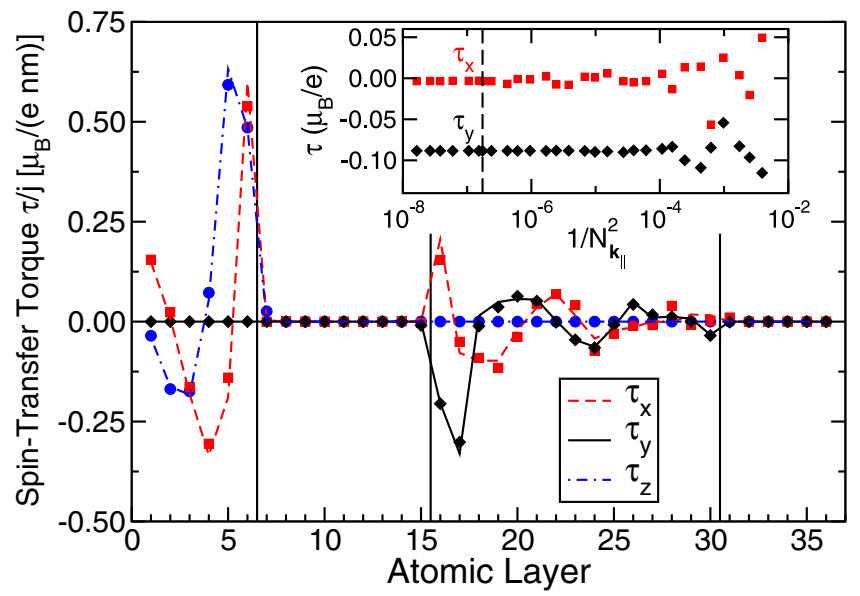

FIG. 7. STT calculated for a spin valve consisting of $\mathrm{Co}$ and $\mathrm{Cu}$ multilayers without SOC. The lines are calculated based on the spin conservation method [14] while the symbols are obtained using the NEM scheme. Inset: total STT on $\mathbf{M}_{2}$ as a function of the $k$ sampling density. The vertical dashed line indicates the final sampling density $(2400 \times 2400)$ adopted.

angles, along the $-z$ direction. Spins injected from the left, oriented perpendicular to this quantization axis, precess in $\mathbf{M}_{2}$. This results in the oscillatory behavior seen for $m_{x}^{\text {ne }}$ and $m_{y}^{\text {ne }}$ in the $\mathbf{M}_{2}$ layer in Fig. 5. In addition, components of $\mathbf{m}^{\text {ne }}$ transverse to $\mathbf{M}_{2}$ decay into the ferromagnetic layer as a result of dephasing [16]. In ferromagnetic Co, the transverse components of $\mathbf{m}^{\text {ne }}$ vanish after propagating about $3 \mathrm{~nm}$ (15 atomic layers) $[13,14,16]$. Eventually, the longitudinal components have the largest magnitude in the ferromagnetic layers, i.e., $m_{y}^{\text {ne }}$ in the left Co layer and $m_{z}^{\text {ne }}$ in the right one.

In spite of their large magnitudes, the longitudinal components of $\mathbf{m}^{\text {ne }}$ do not exert torques on the local magnetization; the smaller transverse components do. The spin torques $\boldsymbol{\tau}$ calculated using Eqs. (13)-(15) are plotted as a function of position in Fig. 7. Reflecting the oscillations in $\mathbf{m}^{\text {ne }}$, the calculated STTs also display oscillations in the Co ferromagnetic layers. The total STT

$$
\boldsymbol{\tau}_{\mathbf{M}_{2}}=\sum_{\mathbf{R} \in \mathbf{M}_{2}} \boldsymbol{\tau}_{\mathbf{R}}
$$

exerted on the right Co layer $\left(\mathbf{M}_{2}\right)$ is plotted in the inset to Fig. 7. It has a large in-plane component in the $-y$ direction and one order of magnitude smaller out-of-plane component in the $-x$ direction. This feature agrees with the spin-transfer picture $[2,3]$ where the conduction electrons polarized by $\mathbf{M}_{1}$ transfer their spin angular momentum to $\mathbf{M}_{2}$ resulting in a STT parallel to $\mathbf{M}_{1}$. Finally, the STTs calculated using the NEM scheme and spin conservation method are in perfect mutual agreement (within the numerical accuracy) and in good agreement [70] with earlier NEM [13] and spin conservation [14] calculations.

\section{SOC-INDUCED STTS IN BALLISTIC Ni DWs}

In this section, we apply the NEM scheme to calculate the spatially resolved STT for Bloch DWs (see Fig. 8 for the profile) in ballistic $\mathrm{Ni}$ in order to obtain a transparent

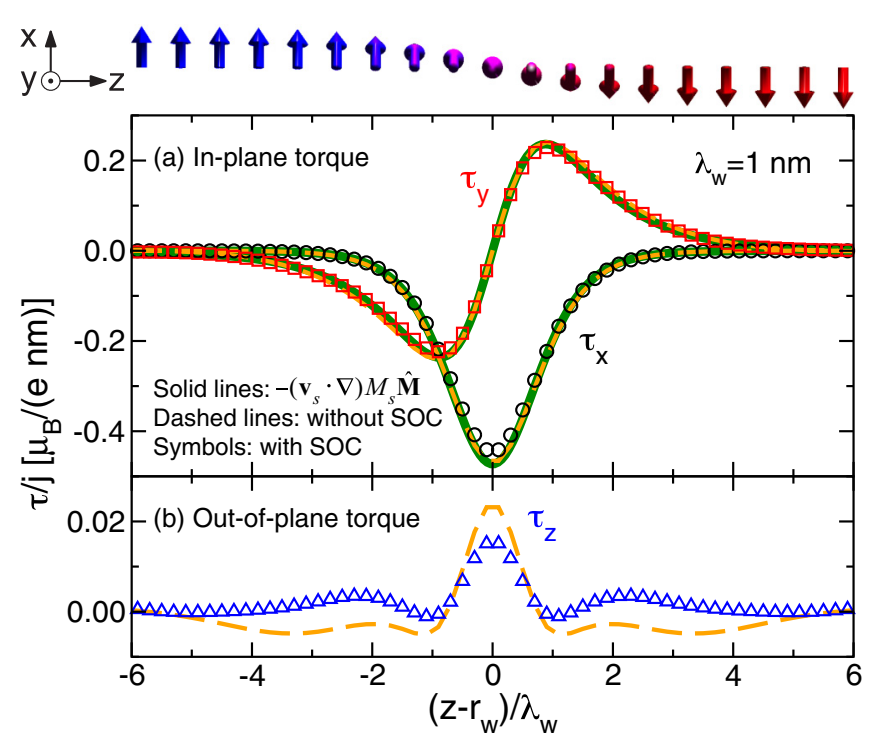

FIG. 8. Calculated STT for a short, clean Ni Bloch DW with $\lambda_{w}=1 \mathrm{~nm}$. The thick solid (green) lines in (a) show the adiabatic form of in-plane torque $-\left(\mathbf{v}_{s} \cdot \nabla\right) M_{s} \hat{\mathbf{M}}$. The dashed (orange) lines in (a) and (b) are the STTs calculated without SOC and the symbols are obtained with SOC. Including SOC gives only slight changes in the calculated STTs. The out-of-plane torque $\tau_{z}$ mainly results from the abrupt variation of the exchange potential in the center of the short DW.

physical picture of the interplay between an electrical current and local magnetization that results from SOC. In particular, we wish to understand the unexpected divergence of $\bar{\beta}$ found in the adiabatic limit with the charge pumping formalism. The numerical details are the same as described in Sec. II B except that a denser $k$ mesh of $2400 \times 2400$ points is used to sample the 2D BZ.

For a very short DW with $\lambda_{w}=1 \mathrm{~nm}$, the in-plane components of the STT are shown in Fig. 8(a) and the out-of-plane component is shown separately in Fig. 8(b). Results without SOC are shown as dashed lines, with SOC as symbols. In the generalized LLG equation, Eq. (1), the expression $-\left(\mathbf{v}_{\mathbf{s}} \cdot \nabla\right) \hat{\mathbf{M}}$ for the in-plane torque comes from spin conservation. In deriving it, it was assumed that conduction electrons can adiabatically follows the orientation of the local magnetization $[73,74]$. At position $\mathbf{r}$, the spin current carried by an electrical current $j$ is given by $\hbar \gamma P j \hat{\mathbf{M}}(\mathbf{r}) /(2 e)$ and the loss of spin current a short distance away from $\mathbf{r}$ corresponds to the STT $-\left(\mathbf{v}_{s} \cdot \nabla\right) M_{s} \hat{\mathbf{M}}(\mathbf{r})$. Using the analytical expression for the onedimensional magnetization profile, the $x$ and $y$ components of the adiabatic torque $\tau^{\text {ad }}(z)=-v_{s} M_{s} d \hat{\mathbf{M}}(z) / d z$ are

$$
\begin{aligned}
\frac{\tau_{x}^{\mathrm{ad}}(z)}{j} & =\frac{\mu_{B} P}{e \lambda_{w}} \operatorname{sech}^{2}\left(\frac{z-r_{w}}{\lambda_{w}}\right), \\
\frac{\tau_{y}^{\mathrm{ad}}(z)}{j} & =-\frac{\mu_{B} P}{e \lambda_{w}} \tanh \left(\frac{z-r_{w}}{\lambda_{w}}\right) \operatorname{sech}\left(\frac{z-r_{w}}{\lambda_{w}}\right) .
\end{aligned}
$$

This adiabatic torque is plotted in Fig. 8(a) as solid green lines where $P=\left(G_{\mathrm{Sh}}^{\uparrow}-G_{\mathrm{Sh}}^{\downarrow}\right) /\left(G_{\mathrm{Sh}}^{\uparrow}+G_{\mathrm{Sh}}^{\downarrow}\right)=-0.48$ is obtained from the spin-resolved Sharvin conductances $G_{\mathrm{Sh}}^{\uparrow}$ and $G_{\mathrm{Sh}}^{\downarrow}$ without SOC. The negative value of $P$ indicates 


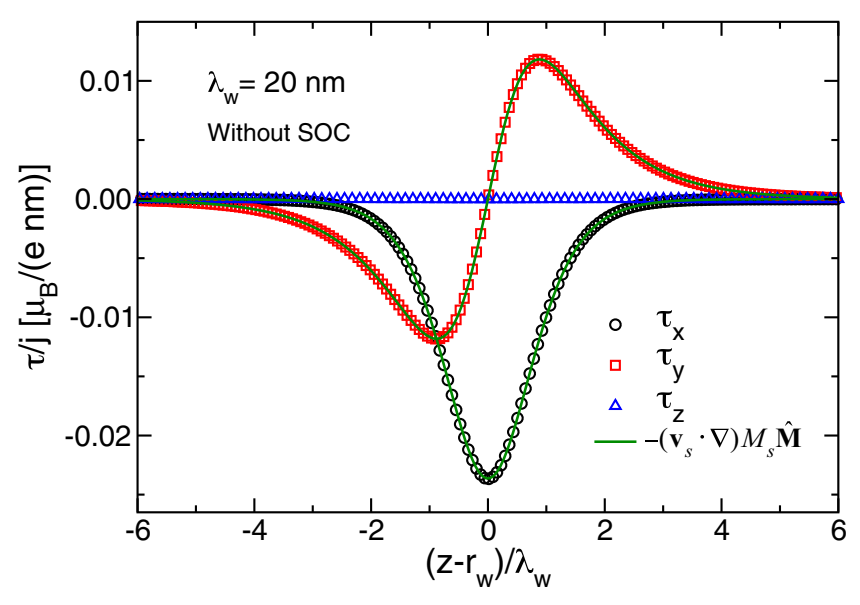

FIG. 9. STTs calculated without SOC for a long, clean Ni Bloch DW with $\lambda_{w}=20 \mathrm{~nm}$. The in-plane torque $\left(\tau_{x}\right.$ and $\left.\tau_{y}\right)$ follows the adiabatic form $-\left(\mathbf{v}_{s} \cdot \nabla\right) M_{s} \hat{\mathbf{M}}$ resulting from spin conservation. The out-of-plane torque $\left(\tau_{z}\right)$ vanishes without SOC in such a long DW.

that the minority-spin channel has more propagating states than the majority-spin channel at the Fermi level; the $s$ band contribution is very similar for both spins while the $d$ contribution is absent from the majority spin channel.

The calculated in-plane STTs shown in Fig. 8(a) are seen to accurately follow the adiabatic form regardless of SOC. The near perfect coincidence of the dashed lines (without SOC) and symbols (with SOC) superposed in Fig. 8(a) on the thick solid lines indicates that the adiabatic form describes the in-plane STT extremely well even in such a short DW. This result is in agreement with a previous calculation for free-electron Stoner-model DWs where the deviation of the in-plane STT from the adiabatic torque was found to be very small [42].

The out-of-plane STT, plotted in Fig. 8(b), is seen to be mainly localized at the DW center and an order of magnitude smaller in size. Since the adiabatic forms in Eqs. (18) do not have an out-of-plane $\left(\tau_{z}\right)$ component, the appearance of such a STT implies a nonadiabaticity of the conduction electrons moving through the DW. In short DWs the out-of-plane torque arises from the nonadiabatic reflection of conduction electrons, especially in the central region of the DW where the magnetization has the largest spatial gradient; including SOC is seen to have relatively little effect. These features are consistent with the observation from Fig. 4 that the calculated out-of-plane parameter $\bar{\beta} \ll 1$ and is not very sensitive to SOC in short DWs.

Without SOC, the nonadiabatic contribution to the $\beta$ torque observed in short DWs decreases as the magnetization gradients in longer walls become smaller; see Fig. 9 for $\lambda_{w}=20 \mathrm{~nm}$. The in-plane components in this case completely follow the adiabatic form $-\left(\mathbf{v}_{s} \cdot \nabla\right) M_{S} \hat{\mathbf{M}}$ and the out-of-plane STT vanishes within the numerical accuracy. Analysis of the conductance shows that only $0.17 \%$ of incoming electrons from the leads are reflected by this $\lambda_{w}=20 \mathrm{~nm} \mathrm{DW}$; the others pass through the DW by adjusting their spins adiabatically.

With SOC included, the electron reflection in long DWs is mainly due to the intrinsic DWR [33,34] and results in out-of-plane torques. The STTs calculated with SOC in two long DWs $\left(\lambda_{w}=20\right.$ and $\left.40 \mathrm{~nm}\right)$ are plotted in Fig. 10, where

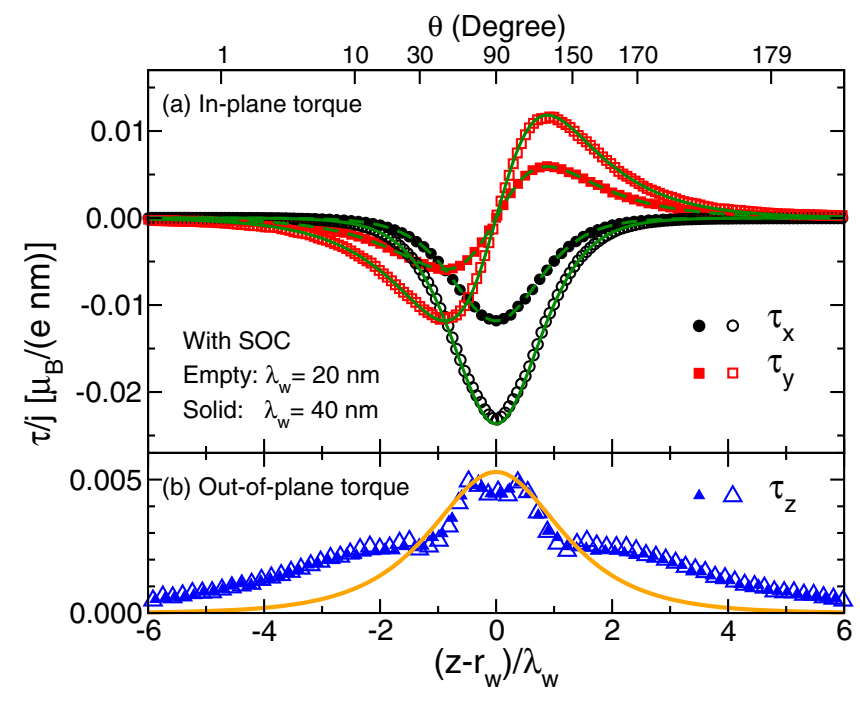

FIG. 10. STTs calculated with SOC for a long, clean Ni Bloch DW with $\lambda_{w}=20$ (empty symbols) and $40 \mathrm{~nm}$ (solid symbols). The in-plane torque (a) is found to be nearly proportional to the magnetization gradient in agreement with the expressions for STT arising from loss of spin current: the solid and dashed lines denote the adiabatic form $-\left(\mathbf{v}_{s} \cdot \nabla\right) M_{s} \hat{\mathbf{M}}$ for $\lambda_{w}=20$ and $40 \mathrm{~nm}$, respectively. The out-of-plane torque (b) arises from the SOC-induced electron reflection due to conduction channel mismatch and is independent of the magnetization gradient. The solid orange line illustrates a constant $\bar{\beta} / \lambda_{w}$ [Eq. (23)] with the value $0.009 \mathrm{~nm}^{-1}$ obtained by integrating the calculated $\tau_{z}(z)$.

the in-plane (a) and out-of-plane (b) components show a different dependence on DW length. The in-plane STT is smaller in the longer DW because it results mainly from the adiabatic spin transfer mechanism [2,3] and is proportional to the magnetization gradient. Note that we plot the STTs with the scaled coordinates $\left(z-r_{w}\right) / \lambda_{w}$ so the integral of the in-plane torque with respect to $z$ is always $-2 v_{s} M_{s} \hat{\mathbf{M}}(-\infty)$, independent of $\lambda_{w}$ though the maximum in-plane torque is proportional to $1 / \lambda_{w}$. The reflection of electrons due to conduction channel mismatch contributes very little to the in-plane torques because only a small number $(1.8 \%)$ of incoming electrons are reflected resulting in a contribution to $\mathbf{m}^{\text {ne }}$ that is much smaller than that due to the adiabatic spin-transfer mechanism. Therefore the in-plane STTs still follow the adiabatic form as we already saw in Fig. 9(a).

The most striking effect of SOC is seen in the out-of-plane torques, which have the same amplitude for both DW lengths at the same scaled position $\left(z-r_{w}\right) / \lambda_{w}$; see Fig. 10(b). Because the expression for the out-of-plane torque in Eq. (1) contains the magnetization gradient (or $1 / \lambda_{w}$ ), a factor $\lambda_{w}$ must be included in the parameters $\beta$ in Eq. (1) and $\bar{\beta}$ in Eq. (5) to reproduce the NEM result shown in Fig. 10(b) of a "constant" local out-of-plane torque. This is consistent with the value of $\bar{\beta}$ calculated with SOC in the adiabatic limit being proportional to $\lambda_{w}$ in Fig. 4.

The width dependence of the calculated torques is reflected in the NEM $\mathbf{m}^{\text {ne }}(z)$ plotted in Fig. 11. The in-plane, adiabatic torque in Fig. 10(a) arises from the out-of-plane component of nonequilibrium magnetization, $m_{z}^{\text {ne }}$, which is shown in 


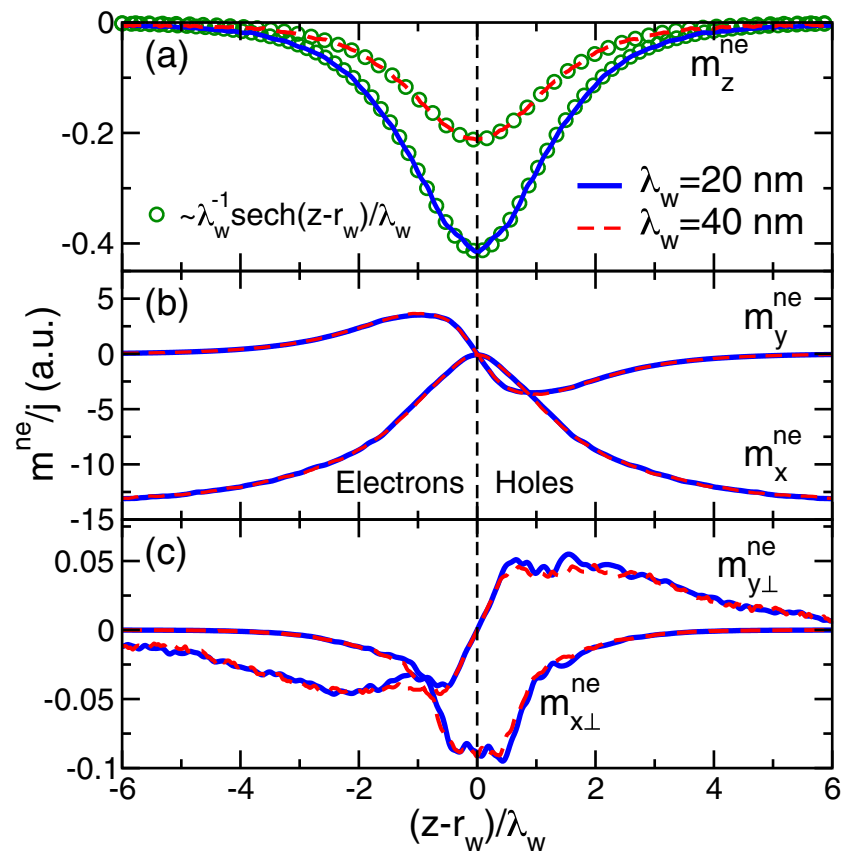

FIG. 11. (a) Calculated out-of-plane NEM $m_{z}^{\text {ne }}$ in long Ni DWs with $\lambda_{w}=20$ and $40 \mathrm{~nm}$. The green circles illustrate the analytical form $\lambda_{w}^{-1} \operatorname{sech}\left(z-r_{w}\right) / \lambda_{w}$ [Eq. (20)]. (b) Calculated in-plane NEM components $m_{x}^{\text {ne }}$ and $m_{y}^{\text {ne }}$ that can be decomposed into components parallel and perpendicular to the local magnetization $\hat{\mathbf{M}}$. (c) The perpendicular components of the calculated in-plane NEM (that are two orders of magnitude smaller than the parallel components) give rise to the width-independent out-of-plane torque $\tau_{z}$ shown in Fig. 10(b).

Fig. 11(a). This component exists because the nonequilibrium magnetization precesses about the local magnetic field, eventually giving rise to the adiabatic torque, i.e.,

$$
\boldsymbol{\tau}^{\mathrm{ad}}=-v_{s} M_{s} \frac{d \hat{\mathbf{M}}}{d z}=-\gamma H^{\mathrm{ex}} \hat{\mathbf{M}} \times m_{z}^{\mathrm{ne}} .
$$

Thus we find

$$
\begin{aligned}
m_{z}^{\mathrm{ne}} & =\hat{\mathbf{M}} \times\left(\hat{\mathbf{M}} \times m_{z}^{\mathrm{ne}}\right)=\frac{v_{s} M_{s}}{\gamma H^{\mathrm{ex}}} \hat{\mathbf{M}} \times \frac{d \hat{\mathbf{M}}}{d z} \\
& =\frac{v_{s} M_{s}}{\gamma H^{\mathrm{ex}}} \frac{1}{\lambda_{w}} \operatorname{sech}\left(\frac{z-r_{w}}{\lambda_{w}}\right),
\end{aligned}
$$

where the position and width dependences are explicitly confirmed by the calculated $m_{z}^{\text {ne }}$ in Fig. 11(a). The inplane nonequilibrium magnetization, that is much larger in magnitude than $m_{z}^{\text {ne }}$, basically follows the local magnetization profile, as shown in Fig. 11(b). Note that $\mathbf{m}^{\text {ne }}$ is made up of electrons and holes accumulated in the left and right halves of the DW, respectively. To investigate the origin of the width-independent out-of-plane torque $\tau_{z}$, we subtract from the calculated $\mathbf{m}^{\text {ne }}$ the parallel component that is aligned with $\hat{\mathbf{M}}$,

$$
\mathbf{m}_{\perp}^{\text {ne }}=(1-\hat{\mathbf{M}} \hat{\mathbf{M}} \cdot) \mathbf{m}^{\text {ne }} .
$$

The $x$ and $y$ components of $\mathbf{m}_{\perp}^{\text {ne }}$ are plotted in Fig. 11(c). Though $m_{x(y) \perp}^{\text {ne }}$ is only about $1 \%$ of the total $m_{x(y)}^{\text {ne }}$, it is responsible for exerting the out-of-plane torque $\tau_{z}$ in the adiabatic limit. Unlike $m_{z}^{\text {ne }}, m_{x(y)}^{\text {ne }}$ and $m_{x(y) \perp}^{\text {ne }}$ do not depend on the DW width which is consistent with the width-independent $\tau_{z}$ in Fig. 10(b). The narrow and high central peak of $\tau_{z}$ comes from $m_{x \perp}^{\text {ne }}$ at the DW center while the broad and weak background arises from the more extended $m_{y \perp}^{\text {ne }}$. The nonanalytical distribution of $m_{x(y) \perp}^{\text {ne }}$ is due to the complex distribution of conduction channels in the $2 \mathrm{D} \mathrm{BZ}$.

The physical picture of SOC-induced out-of-plane STTs that are independent of the DW width can be better understood by comparing them with the torques resulting from the inverse spin-galvanic effect (ISGE) [17,18,75-78]. In systems without inversion symmetry and with SOC (that are frequently modeled using Rashba and Dresselhaus terms), the spin of a propagating state at the Fermi level depends on its momentum and the nonequilibrium magnetization induced by an electrical current is usually not aligned with the local exchange field (local magnetization) [17,18,78]. In such systems, the nonequilibrium magnetization exerts a uniform, "bulk-like" torque on the collinear magnetization.

The SOC-induced out-of-plane STTs shown in Fig. 10(b) have the same bulk-like properties which eventually lead to the $\lambda_{w}$ independence in the adiabatic limit. The scaled position $\left(z-r_{w}\right) / \lambda_{w}$ can be equivalently characterized by a winding angle

$$
\theta(z)=\pi-\cos ^{-1}\left[\tanh \left(\frac{z-r_{w}}{\lambda_{w}}\right)\right],
$$

which rotates from 0 to $\pi$ for a $180^{\circ} \mathrm{DW}$. In a very wide DW, we consider a segment with length $d\left(d \ll \lambda_{w}\right)$, in which the winding angle of the local magnetization varies by the infinitesimal amount $\Delta \theta$; see Fig. 12(a). The anisotropic conduction channels in the $2 \mathrm{D} \mathrm{BZ}$ are schematically shown in Fig. 12(c). The mismatch of conduction channels at $\hat{\mathbf{M}}(\theta)$ (the solid ellipse) and $\hat{\mathbf{M}}(\theta+\Delta \theta)$ (the dashed ellipse) results in reflection of the incoming electronic states shown shaded and consequently a nonequilibrium magnetization $\Delta \mathbf{m}^{\text {ne }}$. Note that in the adiabatic limit $\left(\lambda_{w} \gg d\right), \Delta \mathbf{m}^{\text {ne }}$ and the corresponding torque are both uniform in this segment of the DW and are independent of the length of the segment. In other words, the torque only depends on $\theta$ and $\Delta \theta$ but is independent of the magnetization gradient or of the DW width $\left(\lambda_{w}\right)$, in agreement with the calculated out-of-plane STTs in Fig. 10(b).

A quantitative comparison between Fig. 4 and Fig. 10(b) can be achieved by deriving $\beta(z)$ from the calculated $\tau_{z}(z)$ and performing the integration in Eq. (5) to obtain $\bar{\beta}$, noting that $\tau_{z}(z)$ plotted in Fig. 10(b) is calculated with a constant current density $j$. On the other hand, displacing the DW rigidly with $r_{w}$ in the charge pumping calculations results in relatively large precession and hence large pumped current density at the center of the DW; the further from the center, the less the magnetization changes. Thus we use the relative amplitude of $|d \hat{\mathbf{M}}(z) / d t| \propto \operatorname{sech} \frac{z-r_{w}}{\lambda_{w}}$ as the weight to integrate $\tau_{z}(z)$ and find $\bar{\beta} / \lambda_{w}=0.009 \mathrm{~nm}^{-1}$, which is in very good agreement with the saturated value $0.0086 \mathrm{~nm}^{-1}$ in Fig. 4 obtained from the charge pumping calculations (large open circles).

Our results suggest that the constant- $\beta$ assumption in the literature breaks down for ballistic ferromagnetic DWs in two respects. (i) We find that $\bar{\beta}$ scales with the DW width in the adiabatic limit as shown in Fig. 4 and Fig. 10(b). (ii) In a DW with a fixed width, the calculated out-of-plane STT does not 

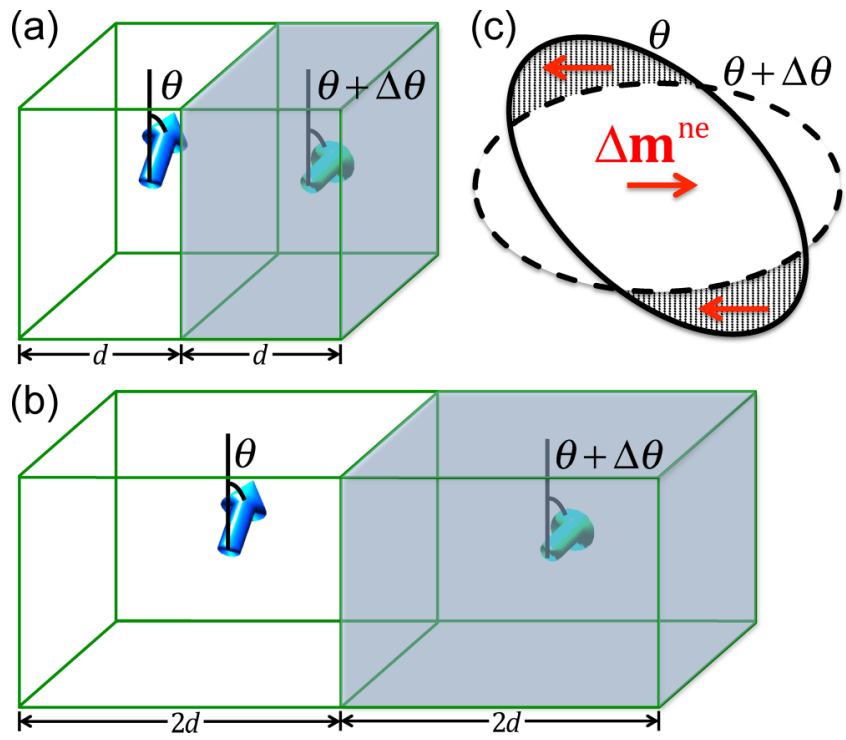

FIG. 12. (a) and (b) Sketch of two small segments of a very wide Bloch DW where the magnetization rotates in the $x y$ plane as a function of $z$, the direction of current flow. The (collinear) magnetization direction of each segment is indicated by an arrow at the center of each segment. The magnetization gradient of the DW in (b) is half of that in (a). (c) Schematic illustration of Fermi surface (FS) projections (ellipses) onto a plane perpendicular to the current, where the conduction channel anisotropy can be represented in terms of the dependence of the FS projection on the local magnetization direction $\hat{\mathbf{M}}(\theta)$. The conduction electrons propagating in the $\theta$ segment (solid ellipse) are filtered when they enter the $\theta+\Delta \theta$ segment with the states in the shaded regions "blocked". Depending on the specific spins of the blocked states, this filtering results in a net nonequilibrium magnetization $\Delta \mathbf{m}^{\text {ne }}$ in the segment $\theta+\Delta \theta$ [shaded volumes in (a) and (b)] regardless of its own width ( $d$ or $2 d$ ). Similarly, another nonequilibrium magnetization can be generated in the segment $\theta$ by the blocked states from entering the segment $\theta+\Delta \theta$. Thus the STT arising from the conduction channel anisotropy has a "bulk-like" property in this segment independent of the global magnetization gradient. The real anisotropic conduction channels are plotted in Figs. 3(a)-3(e) for transport in the [111] direction for fcc Ni.

follow the spatial distribution in the generalized LLG equation (1) with a constant $\beta$. We plotted the constant- $\beta$ form

$$
\frac{\tau_{z}(z)}{j}=\frac{\hbar \gamma P \bar{\beta}}{2 e \lambda_{w}} \operatorname{sech}\left(\frac{z-r_{w}}{\lambda_{w}}\right),
$$

in Fig. 10(b) (the solid orange line) with the calculated $\bar{\beta} / \lambda_{w}=$ $0.009 \mathrm{~nm}^{-1}$. In the central region of the DW $\left(\left|z-r_{w}\right| \leqslant \lambda_{w}\right.$, or $40^{\circ} \leqslant \theta \leqslant 140^{\circ}$ ), Eq. (23) reasonably reproduces the calculated out-of-plane STT. For $\left|z-r_{w}\right|>\lambda_{w}$, Eq. (23) deviates significantly from the calculated $\tau_{z}$. This is because a constant $\beta$ implies an exponential decay of the out-of-plane STT away from the DW center, while $\tau_{z}$ in a real material depends on the conduction channel anisotropy at the Fermi level. The latter may be nonanalytical, something which was recognized in first-principles calculations of magnetocrystalline anisotropy energies [57]. A general form of the current-induced torque to be substituted into the LLG equation has been proposed by
Hals and Brataas [46]

$$
\boldsymbol{\tau}(\mathbf{r})=M_{S} \hat{\mathbf{M}}(\mathbf{r}) \times\left[\int d \mathbf{r}^{\prime} \boldsymbol{\eta}\left(\mathbf{r}, \mathbf{r}^{\prime}\right) \cdot \mathbf{v}_{S}\left(\mathbf{r}^{\prime}\right)\right],
$$

where $\eta\left(\mathbf{r}, \mathbf{r}^{\prime}\right)$ is the so called nonlocal "fieldance" tensor. In the diffusive limit, this can be assumed to be local depending only on the local magnetization and its first-order gradient [46], $\eta(\mathbf{r})=\eta[\hat{\mathbf{M}}(\mathbf{r}), \nabla \hat{\mathbf{M}}(\mathbf{r})]$. In a homogenous system with full rotational symmetry (e.g. without SOC), the leading order expansion of the fieldance tensor gives [46] $\boldsymbol{\eta}=$ $\eta_{1} \nabla \hat{\mathbf{M}}+\eta_{2} \hat{\mathbf{M}} \times \nabla \hat{\mathbf{M}}$ with $\eta_{1}$ and $\eta_{2}$ unknown coefficients. Spin conservation requires $\eta_{2}=1$ and $\eta_{1}$ then corresponds to the parameter $\beta$ in Eq. (1). However, the above derivation is only applicable in the diffusive regime when the mean free path is much shorter than the DW width [41]. In ballistic DWs with infinite mean free path, the mechanism illustrated in Fig. 12 (c) is not described by a local approximation.

In diffusive DWs, disorder allows conduction channels in the shaded regions to scatter to conduction channels in other, allowed areas and the mechanism sketched in Fig. 12(c) is not operative in the adiabatic limit. We have learned that, although the intrinsic DWR vanishes in diffusive DWs with strong disorder scattering, the SOC-induced spin-flip scattering leads to another nonvanishing DWR in the adiabatic limit [64]. By analogy, we might expect other SOC-related mechanisms to emerge in diffusive DWs that contribute to the $\beta$ parameter. It would be interesting to verify to what extent the gradient expansion is applicable. However, this is beyond the scope of the present paper.

\section{CONCLUSIONS}

Using Landauer-Büttiker scattering theory combined with first-principles electronic structure calculations, we have implemented two computational schemes capable of describing spin torques in the presence of spin-orbit interaction, namely, the charge pumping [19] and the nonequilibrium magnetization [24] formalisms. The charge pumping formalism efficiently determines the total current-induced torque in terms of the charge current pumped by a precessing magnetization. We have used this scheme to calculate the DWR and out-ofplane STT parameter $\bar{\beta}$ for ballistic nickel DWs. In addition to the nonadiabatic reflection of conduction electrons by the rapidly varying exchange potentials that leads to a large DWR for very short DWs, an intrinsic DWR arising from SOC dominates the DWR at large DW lengths [33,34]; with SOC included, the out-of-plane STT parameter $\bar{\beta}$ is found to be proportional to the DW length in the adiabatic limit. To understand this unexpected behavior, we implemented the NEM scheme that can be used to calculate position resolved STTs and is physically transparent. We illustrate the NEM scheme using a $\mathrm{Co}|\mathrm{Cu}| \mathrm{Co}$ spin valve as an example. In particular, without SOC the NEM scheme reproduces the STTs obtained for the spin valve from the spatial variation of the spin current combined with spin conservation.

Applying the NEM scheme to calculate position resolved STTs in ballistic Ni DWs, we demonstrate that the inplane STT can be described by the adiabatic form from the generalized LLG equation (1) for both short and long 
DWs, independent of SOC. The position-dependent torques calculated using the NEM scheme allow us to understand the behavior of $\bar{\beta}$ obtained with the charge pumping formalism. For short DWs the nonadiabatic reflection of conduction electrons is the main reason for the out-of-plane torque, independent of SOC. In the adiabatic limit, the SOC induced anisotropic distribution of conduction channels that gives rise to the intrinsic DWR contributes to an out-of-plane torque. This contribution is constant at a given winding angle of a DW such that the parameter $\beta$ in the generalized LLG equation (1) is proportional to the DW length, in quantitative agreement with the result of the charge pumping formalism. The bulk-like out-of-plane torque can be understood by analogy with the spin-orbit torques that result from the ISGE in bulk materials without inversion symmetry. Our results indicate that the constant $\beta$ approximation based upon a gradient expansion in the diffusive regime breaks down for ballistic DWs because of the infinite mean free path.

\section{ACKNOWLEDGMENTS}

We would like to thank Arne Brataas, Kjetil Hals, Yi Liu, Jiang Xiao, Frank Freimuth, Jairo Sinova, Lei Wang, and Pengxiang $\mathrm{Xu}$ for helpful discussions. This work was financially supported by the "Nederlandse Organisatie voor Wetenschappelijk Onderzoek" (NWO) through the research program of "Stichting voor Fundamenteel Onderzoek der Materie" (FOM) and the supercomputer facilities of NWO "Exacte Wetenschappen (Physical Sciences)." It was also partly supported by the Royal Netherlands Academy of Arts and Sciences (KNAW). Z.Y. acknowledges the financial support of the Alexander von Humboldt Foundation.
[1] T. Yang, T. Kimura, and Y. Otani, Giant spin-accumulation signal and pure spin-current-induced reversible magnetization switching, Nat. Phys. 4, 851 (2008).

[2] J. C. Slonczewski, Current-driven excitation of magnetic multilayers, J. Magn. Magn. Mater. 159, L1 (1996).

[3] L. Berger, Emission of spin waves by a magnetic multilayer traversed by a current, Phys. Rev. B 54, 9353 (1996).

[4] A. Brataas, A. D. Kent, and H. Ohno, Current-induced torques in magnetic materials, Nat. Mater. 11, 372 (2012).

[5] J. Åkerman, Toward a universal memory, Science 308, 508 (2005).

[6] M. H. Kryder and C. S. Kim, After hard drives-What comes next? IEEE Trans. Magn. 45, 3406 (2009).

[7] A. Ruotolo, V. Cros, B. Georges, A. Dussaux, J. Grollier, C. Deranlot, R. Guillemet, K. Bouzehouane, S. Fusil, and A. Fert, Phase-locking of magnetic vortices mediated by antivortices, Nat. Nanotechnol. 4, 528 (2009).

[8] A. Slavin, Spin-torque oscillators get in phase, Nat. Nanotechnol. 4, 479 (2009).

[9] S. S. P. Parkin, M. Hayashi, and L. Thomas, Magnetic domainwall racetrack memory, Science 320, 190 (2008).

[10] J. H. Franken, H. J. M. Swagten, and B. Koopmans, Shift registers based on magnetic domain wall ratchets with perpendicular anisotropy, Nat. Nanotechnol. 7, 499 (2012).

[11] X. Waintal, E. B. Myers, P. W. Brouwer, and D. C. Ralph, Role of spin-dependent interface scattering in generating currentinduced torques in magnetic multilayers, Phys. Rev. B 62, 12317 (2000).

[12] G. E. W. Bauer, Yu. V. Nazarov, D. Huertas-Hernando, A. Brataas, K. Xia, and P. J. Kelly, Semiclassical concepts in magnetoelectronics, Mater. Sci. Eng. B 84, 31 (2001).

[13] P. M. Haney, D. Waldron, R. A. Duine, A. S. Núñez, H. Guo, and A. H. MacDonald, Current-induced order parameter dynamics: Microscopic theory applied to $\mathrm{Co} / \mathrm{Cu} / \mathrm{Co}$ spin valves, Phys. Rev. B 76, 024404 (2007).

[14] S. Wang, Y. Xu, and K. Xia, First-principles study of spintransfer torques in layered systems with noncollinear magnetization, Phys. Rev. B 77, 184430 (2008).

[15] K. Xia, P. J. Kelly, G. E. W. Bauer, A. Brataas, and I. Turek, Spin torques in ferromagnetic/normal-metal structures, Phys. Rev. B 65, 220401 (2002).
[16] M. Zwierzycki, Y. Tserkovnyak, P. J. Kelly, A. Brataas, and G. E. W. Bauer, First-principles study of magnetization relaxation enhancement and spin transfer in thin magnetic films, Phys. Rev. B 71, 064420 (2005).

[17] P. M. Haney and M. D. Stiles, Current-Induced Torques in the Presence of Spin-Orbit Coupling, Phys. Rev. Lett. 105, 126602 (2010).

[18] A. Brataas and K. M. D. Hals, Spin-orbit torques in action, Nat. Nanotechnol. 9, 86 (2014).

[19] A. S. Núñez and A. H. MacDonald, Theory of spin transfer phenomena in magnetic metals and semiconductors, Solid State Commun. 139, 31 (2006).

[20] C. Heiliger and M. D. Stiles, Ab Initio Studies of the SpinTransfer Torque in Magnetic Tunnel Junctions, Phys. Rev. Lett. 100, 186805 (2008).

[21] P. M. Haney, H.-W. Lee, K.-J. Lee, A. Manchon, and M. D. Stiles, Current-induced torques and interfacial spin-orbit coupling, Phys. Rev. B 88, 214417 (2013).

[22] F. Freimuth, S. Blügel, and Y. Mokrousov, Spin-orbit torques in $\mathrm{Co} / \mathrm{Pt}(111)$ and $\mathrm{Mn} / \mathrm{W}(001)$ magnetic bilayers from first principles, Phys. Rev. B 90, 174423 (2014).

[23] F. Freimuth, S. Blügel, and Y. Mokrousov, Direct and inverse spin-orbit torques, Phys. Rev. B 92, 064415 (2015).

[24] K. M. D. Hals, A. K. Nguyen, and A. Brataas, Intrinsic Coupling between Current and Domain Wall Motion in (Ga,Mn)As, Phys. Rev. Lett. 102, 256601 (2009).

[25] K. M. D. Hals, A. Brataas, and Y. Tserkovnyak, Scattering theory of charge-current-induced magnetization dynamics, Europhys. Lett. 90, 47002 (2010).

[26] K. Gilmore, I. Garate, A. H. MacDonald, and M. D. Stiles, Firstprinciples calculation of the nonadiabatic spin transfer torque in Ni and Fe, Phys. Rev. B 84, 224412 (2011).

[27] A. Brataas, Y. Tserkovnyak, G. E. W. Bauer, and P. J. Kelly, Spin pumping and spin transfer, in Spin Current, Semiconductor Science and Technology, Vol. 17, edited by S. Maekawa, S. O. Valenzuela, E. Saitoh, and T. Kimura (Oxford University Press, Oxford, 2012) Chap. 8, pp. 87-135.

[28] C. A. Akosa, W.-S. Kim, A. Bisig, M. Kläui, K.-J. Lee, and A. Manchon, Role of spin diffusion in current-induced domain wall motion for disordered ferromagnets, Phys. Rev. B 91, 094411 (2015). 
[29] T. Weindler, H. G. Bauer, R. Islinger, B. Boehm, J.-Y. Chauleau, and C. H. Back, Magnetic Damping: Domain Wall Dynamics versus Local Ferromagnetic Resonance, Phys. Rev. Lett. 113, 237204 (2014).

[30] Z. Yuan, K. M. D. Hals, Y. Liu, Anton A. Starikov, A. Brataas, and P. J. Kelly, Gilbert Damping in Noncollinear Ferromagnets, Phys. Rev. Lett. 113, 266603 (2014).

[31] K. Xia, M. Zwierzycki, M. Talanana, P. J. Kelly, and G. E. W. Bauer, First-principles scattering matrices for spin-transport, Phys. Rev. B 73, 064420 (2006).

[32] A. A. Starikov, P. J. Kelly, A. Brataas, Y. Tserkovnyak, and G. E. W. Bauer, Unified First-Principles Study of Gilbert Damping, Spin-Flip Diffusion and Resistivity in Transition Metal Alloys, Phys. Rev. Lett. 105, 236601 (2010).

[33] A. K. Nguyen, R. V. Shchelushkin, and A. Brataas, Intrinsic Domain-Wall Resistance in Ferromagnetic Semiconductors, Phys. Rev. Lett. 97, 136603 (2006).

[34] R. Oszwałdowski, J. A. Majewski, and T. Dietl, Influence of band structure effects on domain-wall resistance in diluted ferromagnetic semiconductors, Phys. Rev. B 74, 153310 (2006).

[35] S. Zhang and Z. Li, Roles of Nonequilibrium Conduction Electrons on the Magnetization Dynamics of Ferromagnets, Phys. Rev. Lett. 93, 127204 (2004).

[36] A. Thiaville, Y. Nakatani, J. Miltat, and Y. Suzuki, Micromagnetic understanding of current-driven domain wall motion in patterned nanowires, Europhys. Lett. 69, 990 (2005).

[37] Y. Tserkovnyak, H. J. Skadsem, A. Brataas, and G. E. W Bauer, Current-induced magnetization dynamics in disordered itinerant ferromagnets, Phys. Rev. B 74, 144405 (2006).

[38] Y. Tserkovnyak, A. Brataas, and G. E. W Bauer, Theory of current-driven magnetization dynamics in inhomogeneous ferromagnets, J. Magn. Magn. Mater. 320, 1282 (2008).

[39] A. Brataas, Y. Tserkovnyak, and G. E. W. Bauer, Magnetization dissipation in ferromagnets from scattering theory, Phys. Rev. B 84, 054416 (2011).

[40] H. Y. Yuan, Z. Yuan, K. Xia, and X. R. Wang, Influence of nonlocal damping on the field-driven domain wall motion, arXiv:1604.07971.

[41] H. Kohno, G. Tatara, and J. Shibata, Microscopic calculation of spin torques in disordered ferromagnets, J. Phys. Soc. Jpn. 75, 113706 (2006).

[42] J. Xiao, A. Zangwill, and M. D. Stiles, Spin-transfer torque for continuously variable magnetization, Phys. Rev. B 73, 054428 (2006).

[43] A. K. Nguyen, H. J. Skadsem, and A. Brataas, Giant CurrentDriven Domain Wall Mobility in (Ga,Mn)As, Phys. Rev. Lett. 98, 146602 (2007).

[44] S. Bohlens and D. Pfannkuche, Width Dependence of the Nonadiabatic Spin-Transfer Torque in Narrow Domain Walls, Phys. Rev. Lett. 105, 177201 (2010).

[45] Z. Yuan, S. Wang, and K. Xia, Thermal spin-transfer torques on magnetic domain walls, Solid State Commun. 150, 548 (2010).

[46] K. M. D. Hals and A. Brataas, Phenomenology of currentinduced spin-orbit torques, Phys. Rev. B 88, 085423 (2013); Spin-orbit torques and anisotropic magnetization damping in Skyrmion crystals, ibid. 89, 064426 (2014).

[47] N. L. Schryer and L. R. Walker, The motion of $180^{\circ}$ domain walls in uniform de magnetic fields, J. Appl. Phys. 45, 5406 (1974).
[48] R. A. Duine, Effects of nonadiabaticity on the voltage generated by a moving domain wall, Phys. Rev. B 79, 014407 (2009).

[49] S. R. de Groot, Thermodynamics of Irreversible Processes (North Holland, Amsterdam, 1952).

[50] Y. Tserkovnyak, A. Brataas, G. E. W. Bauer, and B. I. Halperin, Nonlocal magnetization dynamics in ferromagnetic heterostructures, Rev. Mod. Phys. 77, 1375 (2005).

[51] O. K. Andersen, Linear methods in band theory, Phys. Rev. B 12, 3060 (1975).

[52] O. K. Andersen, Z. Pawlowska, and O. Jepsen, Illustration of the linear-muffin-tin-orbital tight-binding representation: Compact orbitals and charge density in Si, Phys. Rev. B 34, 5253 (1986).

[53] U. von Barth and L. Hedin, A local exchange-correlation potential for the spin-polarized case: I, J. Phys. C: Solid State Phys. 5, 1629 (1972).

[54] O. K. Andersen, O. Jepsen, and D. Glötzel, Canonical description of the band structures of metals, in Highlights of Condensed Matter Theory, International School of Physics "Enrico Fermi", Varenna, Italy, edited by F. Bassani, F. Fumi, and M. P. Tosi (North-Holland, Amsterdam, 1985), pp. 59-176.

[55] T. Ando, Quantum point contacts in magnetic fields, Phys. Rev. B 44, 8017 (1991).

[56] Y. Liu, Z. Yuan, R. J. H. Wesselink, A. A. Starikov, M. van Schilfgaarde, and P. J. Kelly, Direct method for calculating temperature-dependent transport properties, Phys. Rev. B 91, 220405(R) (2015).

[57] G. H. O. Daalderop, P. J. Kelly, and M. F. H. Schuurmans, First-principles calculation of the magnetocrystalline anisotropy energy of iron, cobalt and nickel, Phys. Rev. B 41, 11919 (1990); Magnetic anisotropy of a free-standing Co monolayer and of multilayers which contain Co monolayers, ibid. 50, 9989 (1994).

[58] S. Datta, Electronic Transport in Mesoscopic Systems (Cambridge University Press, Cambridge, 1995).

[59] G. G. Cabrera and L. M. Falicov, Theory of the residual resistivity of Bloch walls I: Paramagnetic effects, Phys. Status Solidi B 61, 539 (1974).

[60] J. B. A. N. van Hoof, K. M. Schep, A. Brataas, G. E. W. Bauer, and P. J. Kelly, Ballistic electron transport through magnetic domain walls, Phys. Rev. B 59, 138 (1999).

[61] J. Kudrnovský, V. Drchal, I. Turek, P. Středa, and P. Bruno, Magnetoresistance in domain walls: Effect of randomness, Surf. Sci. 482-485, 1107 (2001).

[62] K. M. Schep, P. J. Kelly, and G. E. W. Bauer, Giant Magnetoresistance without Defect Scattering, Phys. Rev. Lett. 74, 586 (1995);Ballistic transport and electronic structure, Phys. Rev. B 57, 8907 (1998).

[63] P. X. Xu, K. Xia, M. Zwierzycki, M. Talanana, and P. J. Kelly, Orientation-Dependent Transparency of Metallic Interfaces, Phys. Rev. Lett. 96, 176602 (2006).

[64] Z. Yuan, Y. Liu, A. A. Starikov, P. J. Kelly, and A. Brataas, SpinOrbit-Coupling-Induced Domain-Wall Resistance in Diffusive Ferromagnets, Phys. Rev. Lett. 109, 267201 (2012).

[65] P. M. Haney, R. A. Duine, A. S. Núñez, and A. H. MacDonald, Current-induced torques in magnetic metals: Beyond spintransfer, J. Magn. Magn. Mater. 320, 1300 (2008).

[66] C. Heiliger, M. Czerner, B. Y. Yavorsky, I. Mertig, and M. D. Stiles, Implementation of a nonequilibrium Green's function 
method to calculate spin-transfer torque, J. Appl. Phys. 103, 07A709 (2008).

[67] Applying the bias to the chemical potential on the left (right) electrode would lead to an artificial accumulation of electrons (holes) in the scattering region, which should not exist in metallic systems with strong screening. Neglecting such screening does not change the conductance but yields spurious results for local quantities such as the chemical potential, nonequilibrium magnetization, spin torque, etc. In ballistic systems, the artificial accumulation of charge is usually minimized by applying the bias to the chemical potentials of the left and right leads as $\varepsilon_{\mathrm{F}}+e V_{b} / 2$ and $\varepsilon_{\mathrm{F}}-e V_{b} / 2$, respectively, as we do in this paper. A more rigorous treatment of screening is necessary for diffusive systems, in particular when SOC is included (R. J. H. Wesselink, Z. Yuan, Y. Liu, A. Brataas, and P. J. Kelly, unpublished ); it has negligible effect on the results presented in this paper.

[68] O. Gunnarsson, Band model for magnetism of transition metals in the spin-density-functional formalism, J. Phys. F: Metal Phys. 6, 587 (1976).

[69] D. D. Koelling and B. N. Harmon, A technique for relativistic spin-polarised calculations, J. Phys. C: Sol. State Phys. 10, 3107 (1977).

[70] The results shown in Fig. 5 of Ref. [13] are for transport along a (001) oriented system. Our results can be compared in detail to those shown in Fig. 4(a) of Ref. [14].
[71] P. E. Blöchl, O. Jepsen, and O. K. Andersen, Improved tetrahedron method for Brillouin-zone integrations, Phys. Rev. B 49, 16223 (1994).

[72] K. Xia, P. J. Kelly, G. E. W. Bauer, I. Turek, J. Kudrnovský, and V. Drchal, Interface resistance of disordered magnetic multilayers, Phys. Rev. B 63, 064407 (2001).

[73] Ya. B. Bazaliy, B. A. Jones, and S.-C. Zhang, Modification of the Landau-Lifshitz equation in the presence of a spin-polarized current in colossal- and giant-magnetoresistive materials, Phys. Rev. B 57, R3213(R) (1998).

[74] G. Tatara and H. Kohno, Theory of Current-Driven Domain Wall Motion: Spin Transfer versus Momentum Transfer, Phys. Rev. Lett. 92, 086601 (2004).

[75] V. M. Edelstein, Spin polarization of conduction electrons induced by electric current in two-dimensional asymmetric electron systems, Solid State Commun. 73, 233 (1990).

[76] A. Manchon and S. Zhang, Theory of nonequilibrium intrinsic spin torque in a single nanomagnet, Phys. Rev. B 78, 212405 (2008); Theory of spin torque due to spin-orbit coupling, ibid. 79, 094422 (2009).

[77] I. Garate and A. H. MacDonald, Influence of a transport current on magnetic anisotropy in gyrotropic ferromagnets, Phys. Rev. B 80, 134403 (2009).

[78] J. Sinova, S. O. Valenzuela, J. Wunderlich, C. H. Back, and J. Jungwirth, Spin Hall effects, Rev. Mod. Phys. 87, 1213 (2015). 